\title{
Ferroportin 3 is a dual-targeted mitochondrial/chloroplast iron exporter necessary for iron homeostasis in Arabidopsis
}

Leah J. Kim ${ }^{1}$, Kaitlyn M. Tsuyuki ${ }^{1}$, Fengling $\mathrm{Hu}^{1, \dagger}$, Emily Y. Park ${ }^{1, \dagger}$, Jingwen Zhang ${ }^{1, \dagger}$, Jennifer Gallegos Iraheta ${ }^{1}$, Ju-Chen Chia $^{2}$, Rong Huang ${ }^{3}$, Avery E. Tucker ${ }^{1}$, Madeline Clyne ${ }^{1}$, Claire Castellano ${ }^{1}$, Angie Kim ${ }^{1}$, Daniel D. Chung ${ }^{1}$, Christopher T. DaVeiga ${ }^{1}$, Elizabeth M. Parsons $^{1}$, Olena K. Vatamaniuk ${ }^{2}$, Jeeyon Jeong ${ }^{1, *}$

${ }^{1}$ Department of Biology, Amherst College, Amherst, Massachusetts 01002

${ }^{2}$ Soil and Crop Sciences Section, School of Integrative Plant Science, Cornell University, Ithaca, New York 14853

${ }^{3}$ Cornell High Energy Synchrotron Source, Ithaca, New York 14853

Running title: A mitochondrial and chloroplast ferroportin in Arabidopsis

Keywords: Iron, Ferroportin, Mitochondria, Chloroplast, Arabidopsis thaliana, Transport

*Author for contact: jjeong@amherst.edu

${ }^{\dagger}$ These authors have equally contributed to the manuscript.

J.J. conceived the idea and supervised the study, J.J wrote the article with contributions of L.K., K.T., F.H., E.Y.P., J.Z., and J.G.; Microscopy experiments were conducted by L.K., E.Y.P., and J.J; L.K., F.H., J.Z., J.G., M.C., and C.C. performed the yeast experiments; L.K., J.G., and E.M.P. conducted the GUS assays; L.K., K.T., E.Y.P., F. H., and J.Z., conducted gene expression analyses; L.K. and C.D. carried out the plant growth assays; F.H., A.T., K.T., A.K., 
and D.C. performed cloning and generated transgenic lines; L.K. isolated chloroplasts; L.K. and K.T. isolated mitochondria; L.K. and K.T. conducted Western blots and ICP-MS; L.K and K.T. analyzed TEM data; J-C.C. and R.H. conducted SXRF imaging; O.K.V. supervised the SXRF experiments; all authors read and approved the manuscript.

Significance statement: Iron homeostasis must be tightly controlled in the mitochondria and chloroplasts, but iron trafficking in these organelles is not fully understood. Our work suggests that FPN3 is an iron exporter required for maintaining proper iron levels in mitochondria and chloroplasts. Furthermore, FPN3 is necessary for the optimal growth and normal mitochondrial ultrastructure under iron deficiency. This study reveals the physiological role of FPN3 and advances our understanding of iron regulation in mitochondria and chloroplasts. 


\section{ABSTRACT}

Mitochondria and chloroplasts are organelles with high iron demand that are particularly susceptible to iron-induced oxidative stress. Despite the necessity of strict iron regulation in these organelles, much remains unknown about mitochondrial and chloroplast iron transport in plants. Here, we propose that Arabidopsis Ferroportin 3 (FPN3) is an iron exporter dual-targeted to mitochondria and chloroplasts. FPN3 is expressed in shoots regardless of iron conditions, but its transcripts accumulate under iron deficiency in roots. fpn3 mutants cannot grow as well as wild type under iron-deficient conditions and shoot iron levels are reduced in fpn 3 mutants compared to wild type. ICP-MS measurements show that iron levels in the mitochondria and chloroplasts are increased relative to wild type, consistent with the proposed role of FPN3 as a mitochondrial/plastid iron exporter. In iron deficient fpn3 mutants, abnormal mitochondrial ultrastructure was observed, whereas chloroplast ultrastructure was not affected, implying that FPN3 plays a critical role in the mitochondria. Overall, our study suggests that FPN3 is essential for optimal iron homeostasis. 


\section{INTRODUCTION}

Iron serves as a critical redox cofactor in vital cellular processes. Nevertheless, excess or improperly regulated iron can cause deleterious effects by generating hydroxyl radicals via the Fenton reaction (Halliwell and Gutteridge, 1992). Therefore, iron homeostasis must be tightly maintained in all organisms, including plants. As photosynthetic organisms, plants use iron as an essential cofactor in both respiration and photosynthesis. At the same time, plant cells must regulate iron to ensure adequate supply while avoiding oxidative stress (Shcolnick and Keren, 2006). Despite being an abundant element in the soil, iron is one of the most limiting nutrients for plant growth - it has extremely low bioavailability under aerobic conditions at neutral or alkaline pH (Marschner, 2012; Colombo et al., 2014). Iron homeostasis in plants is of particular interest, because understanding its mechanisms will provide insights to improving agriculture and human health (Vasconcelos et al., 2017).

Dicots acquire iron by a reduction-based mechanism that is induced under iron deficiency (Jeong et al., 2017; Connorton et al., 2017; Kobayashi et al., 2018; Brumbarova et al., 2015). Ferric chelates in the rhizosphere are solubilized by the release of protons (Santi and Schmidt, 2009) and reduced to ferrous iron by FERRIC REDUCTASE OXIDASE 2 (FRO2) (Robinson et al., 1999). Coumarins released from iron-deficient roots are considered to aid this process as well (Clemens and Weber, 2016; Schmidt et al., 2014; Fourcroy et al., 2014). Ferrous iron is then transported into the root via IRON-REGULATED TRANSPORTER 1 (IRT1) (Connolly et al., 2002; Vert et al., 2002; Varotto et al., 2002). Once iron reaches the root vasculature, it is loaded into the xylem by FERROPORTIN 1 (FPN1) (Morrissey et al., 2009) and chelated with citrate, which is transported into the xylem by the FERRIC REDUCTASE DEFECTIVE 3 (FRD3) transporter (Durrett et al., 2007). The iron-citrate complex is then translocated to shoots. For 
lateral translocation in shoots, iron-nicotianamine (NA) complexes are formed and translocated from leaves to seeds via the phloem by YELLOW STRIPE-LIKE (YSL) family members (DiDonato et al., 2004; Schaaf et al., 2005; Waters et al., 2006). Two YSL members, YSL1 and YSL3, were shown to regulate long-distance iron-deficiency signals from shoots (Kumar et al., 2017) and are responsible for loading iron into the seeds (Le Jean et al., 2005; Waters et al., 2006). Oligopeptide transporter 3 (OPT3) also plays a role in transmitting shoot-to-root iron signals and regulates the redistribution of iron from source to sink tissues, such as from old leaves to seeds or to developing tissues (Mendoza-Cózatl et al., 2014; Zhai et al., 2014; Stacey et al., 2008).

In addition to iron acquisition and its distribution between tissues, iron trafficking across subcellular compartments is crucial for proper iron homeostasis. In particular, chloroplasts and mitochondria need a substantial amount of iron; many components of the photosynthetic and respiratory electron transport chains use iron as a cofactor. Fe-S cluster assembly occurs in these organelles and heme biosynthesis occurs in plastids (Masuda et al., 2003; Tanaka et al., 2011). Proteins involved in the last stage of heme biosynthesis are also present in mitochondria (Balk and Schaedler, 2014). Chloroplasts are the most iron-rich organelle in plant cells and accounts for $60-80 \%$ of iron found in a leaf cell (Terry and Low, 1982; Shikanai et al., 2003). Meanwhile, in the mitochondria, iron is the major micronutrient present with a molar ratio of 26:8:6:1 for Fe:Zn:Cu:Mn (Tan et al., 2010). Mitochondria and chloroplasts are also highly susceptible to oxidative stress due to reactive oxygen species (ROS) generated by the electron transport chain. It has been shown that the iron sequestering protein ferritin is present in mitochondria and plastids, and helps to prevent iron-induced oxidative stress (Briat et al., 2010; Zancani et al., 2004; Tarantino, Casagrande, et al., 2010). In the mitochondria, frataxin, which is involved in 
Fe-S cluster and heme biogenesis, also plays a role in protection against iron-induced oxidative stress (Gomez-Casati et al., 2018).

Iron transport in mitochondria and chloroplasts is not as well understood as the mechanisms of iron acquisition in the roots, but molecular and physiological studies have been gradually contributing to understanding iron regulation in chloroplasts and mitochondria. In chloroplasts, physiological studies suggest that ferric chelates move across the outer membrane (Bughio et al., 1997; Solti et al., 2012; Müller et al., 2019), but ferrous iron is imported into chloroplasts across the inner membrane (Bughio et al., 1997; Shingles et al., 2002). The ferric chelate reductase, FRO7, reduces iron for chloroplast iron acquisition (Jeong et al., 2008), and PERMEASE IN CHLOROPLAST 1 (PIC1) mediates iron transport into chloroplasts via interaction with NiCo (Duy et al., 2007; Duy et al., 2011). A recent study in Brassica napus proposes that NiCo may also be involved in iron sensing or iron release from chloroplasts (Pham et al., 2020). The Arabidopsis MitoFerrinLike1 (Mfl1) has also been reported to import iron into chloroplasts (Tarantino et al., 2011). In addition, ATP-binding cassette transporters, ABCI10, NAP14/ABCI11, and ABCI12, may also contribute to iron uptake into chloroplasts (ShimoniShor et al., 2010; Voith von Voithenberg et al., 2019). YSL4 and YSL6 have been reported to efflux iron-NA complexes from the chloroplasts (Divol et al., 2013). However, both transporters were also identified in the tonoplast proteome (Jaquinod et al., 2007) and were targeted to the vacuolar and intracellular membranes (Conte et al., 2013). ZmFRD4 was reported as a potential thylakoid iron importer in maize, although direct evidence for iron transport remains to be found (Zhang et al., 2017). 
In the mitochondria, a mitoferrin ortholog from rice, MITOCHONDRIAL IRON

TRANSPORTER 1 (MIT1), was identified as a mitochondrial iron importer (Bashir et al., 2011).

Two Arabidopsis mitoferrin orthologs, MIT1 and MIT2, have recently been reported as mitochondrial iron importers that mediate cellular iron homeostasis and are essential for embryogenesis (Jain et al., 2019). A reduction-based mechanism might be involved in moving iron in and out of mitochondria, because ferric chelate reductases, FRO3 and FRO8, are localized to the mitochondria in Arabidopsis (Jeong and Connolly, 2009; Heazlewood et al., 2004). The two mitochondrial FROs are likely to play non-overlapping roles, as FRO3 is induced by iron deficiency, whereas FRO8 is not iron-regulated (Jeong and Connolly, 2009; Mukherjee et al., 2006).

In this study, we investigated an Arabidopsis FPN family member, FPN3/IREG3. FPNs, also known as solute carrier (SCL) group 40A1 and IRON REGULATED (IREG) transporters, efflux iron from the cytoplasm (Drakesmith et al., 2015). In animals, FPNs are found in cells that play critical roles in supplying iron, such as intestinal cells. While animal species have only one FPN, multiple FPN paralogs are present within most plant species. In Arabidopsis, three FPN/IREG family members were identified based on phylogenetic analysis (Schaaf et al., 2006). FPN1/IREG1 (At2g38460) and FPN2/IREG2 (At5g03570) share 77 \% identity, whereas FPN3/IREG3 (At5g26820) shares about 20\% identity with FPN1 and FPN2 (Figure 1). FPN1/IREG1 localizes to the plasma membrane of cells in the stele, and FPN2/IREG2 is localized on the vacuolar membrane to buffer cellular metal influx (Morrissey et al., 2009). Previously, FPN3/IREG3 was reported as Multiple Antibiotic Resistance 1 (MAR1), a chloroplast protein that allows antibiotics to opportunistically enter chloroplasts (Conte et al., 2009). While it was speculated that MAR1 may be involved in iron homeostasis, its potential 
role in iron regulation and its physiological function were not previously studied. Here, we report that FPN3 is dual-targeted to mitochondria and chloroplasts, and provide results suggesting that FPN3 exports iron from these organelles. Furthermore, our study provides key evidence indicating that FPN3 plays a critical role in iron homeostasis and its function is important in the mitochondria as evidenced by the drastic morphological changes in fpn 3 mitochondria under iron deficient conditions.

\section{RESULTS}

\section{Iron binding and transport residues of human FPN are conserved in Arabidopsis FPN3}

To investigate the potential role of FPN3 in iron homeostasis, we first examined if conserved iron binding and transport residues of other FPN members are present in FPN3. Structural modeling and in vitro assays with mutated variants of human FPN identified amino acids that are critical for iron binding and transport (Bonaccorsi di Patti et al., 2014; Taniguchi et al., 2015). These residues were highly conserved across FPN orthologs in human, mouse, zebrafish, frog, bacteria, and Arabidopsis FPN paralogs (Taniguchi et al., 2015). For example, two Asp residues

of the human FPN, Asp39 and Asp181, that are essential for iron transport (Bonaccorsi di Patti et al., 2014; Taniguchi et al., 2015) were highly conserved in FPN3 as indicated by presence of the Glu, which is synonymous to Asp, and Asp residues in their corresponding positions (Figure 1).

Two other iron binding/transport residues of HsFPN, Asp325 and Asn174, were conserved in

FPN3 as well (Figure 1). Arg466 of HsFPN, another amino acid that affected iron efflux

(Bonaccorsi di Patti et al., 2014; Taniguchi et al., 2015), was not preserved in FPN3 (Figure 1). However, this Arg is not part of the metal binding site (Bonaccorsi di Patti et al., 2014;

Taniguchi et al., 2015) and the Gly found in this position was conserved among the bacterial and Arabidopsis FPNs. Overall, the conserved iron binding/transport residues suggest that FPN3 is 
highly likely to transport ferrous iron, which is a major substrate of FPN orthologs(Drakesmith et al., 2015; Taniguchi et al., 2015).

\section{Heterologously expressed $F P N 3$ exports iron from mitochondria in yeast}

Multiple chloroplast proteins have been targeted to the mitochondria when expressed in fungi (Versaw and Harrison, 2002; Jeong et al., 2008; Hurt et al., 1986; Pfaller et al., 1989; Brink et al., 1994). Based on the prior report on MAR1 that indicated chloroplast localization (Conte et al., 2009) and the predicted transit peptides that suggested targeting to chloroplasts or mitochondria (Schwacke et al., 2003), we tested if FPN3 would be able to complement mitochondrial iron transporter mutants of Saccharomyces cerevisiae. After verifying that FPN3 is localized to mitochondria using Western blots with mitochondrial fractions of yeast cells expressing FPN3-FLAG (Supplementary Figure 1), we expressed FPN3 in $\triangle m m t 1 / 2$, which lack mitochondrial iron exporters. Previous studies showed that $\Delta m m t 1 / 2 \mathrm{did}$ not have a strong phenotype, but the deletion of $M M T 1 / 2$ in a $\Delta c c c 1$ background, which lacks a vacuolar iron importer, resulted in a slightly decreased sensitivity to high iron compared to $\Delta c c c 1$ (Li et al., 2014). Additionally, $\Delta c c c 1 \Delta m m t 1 / 2$ cells expressing $M M T 1 / 2$ are highly sensitive to high iron due to increased cytosolic iron (Li et al., 2014). In the present study, we expressed FPN3 in $\Delta c c c 1 \Delta m m t 1 / 2$ under high iron conditions to test if phenotypes similar to $\Delta c c c 1 \Delta m m t 1 / 2$ cells expressing MMT1/2 would arise. Consistent with observations by Li et al. (2014), the expression of $M M T 1 / 2$ resulted in reduced growth in high iron (Figure 2A, 2B). The growth of $\Delta c c c 1 \Delta m m t 1 / 2$ expressing FPN3 was decreased in high iron and approximated that of $\Delta c c c 1 \Delta m m t 1 / 2$ expressing $M M T 1 / 2$ (Figure 2A, 2B). Thus, our high iron growth assay results from both plates and liquid cultures suggest that FPN3 exports iron from the mitochondria (Figure 2A, 2B). 
We also expressed FPN3 in the $\triangle m r s 3 \Delta m r s 4$ yeast mutant, which lacks the mitochondrial iron importers, Mrs3 and Mrs4, to test if FPN3 is likely to import iron into mitochondria. The $\Delta m r s 3 \Delta m r s 4$ mutant does not have a strong iron phenotype, but exhibits increased sensitivity to oxidative stress compared to wild type cells (Mühlenhoff et al., 2003; Foury and Roganti, 2002). Expression of FPN3 in $\triangle m r s 3 \triangle m r s 4$ did not rescue the oxidative stress sensitivity of $\triangle m r s 3 \Delta m r s 4$ (Supplementary Figure 2), which would be expected if FPN3 were a mitochondrial iron importer. By contrast, FPN3 expression exacerbated the phenotype, consistent with that of cells expressing MMT1/2 (Supplementary Figure 2), indicating that FPN3 is not likely to be importing iron into the mitochondria.

Based on the results from $\Delta c c c 1 \Delta m m t 1 / 2$ and $\Delta m r s 3 \Delta m r s 4$ cells expressing $F P N 3$ or $M M T 1 / 2$, we hypothesized that FPN3 is a mitochondrial iron exporter. If FPN3 is exporting iron from the mitochondria, mitochondrial iron levels should be lower in cells overexpressing FPN3. To test this hypothesis, we measured the activity of aconitase in mitochondria to indirectly assess mitochondrial iron levels. Aconitase is a Fe-S enzyme involved in the tricarboxylic acid cycle, and its activity correlates with the availability of mitochondrial iron. For example, decreased aconitase activity has been detected in the yeast $\Delta m r s 3 / 4$ cells and rice plants defective in mitochondrial iron import (Foury and Roganti, 2002; Bashir et al., 2011). Although aconitase is present in the mitochondria and the cytosol, mitochondrial aconitase accounts for most of the cellular aconitase activity in yeast, and yeast cytosolic aconitase is not involved in regulating iron metabolism unlike in mammalian cells (Regev-Rudzki et al., 2005). Aconitase activity of cells expressing FPN3 was similar to cells expressing MMT1/2 and exhibited about 30\% lower 
activity than that of the negative control with an empty vector (Figure 2C), suggesting that FPN3 is exporting iron from the mitochondria.

As an alternative approach to testing mitochondrial iron export by FPN3, we examined if cytosolic iron levels increased in cells expressing FPN3. We co-transformed FPN3 or control plasmids along with a plasmid that expresses the bacterial gentisate 1,2-dioxygenase (GDO) in the yeast cytoplasm (Li et al., 2012) and measured GDO activity. GDO uses iron as a cofactor and the activity of cytosolic GDO (c-GDO), which has been confirmed to localize to the cytoplasm in yeast, correlates with the amount of cytosolic iron. Thus, c-GDO can serve as an indicator of cytosolic iron levels in yeast (Li et al., 2012; Li et al., 2014). We detected significantly higher c-GDO activity in cells expressing FPN3 or $M M T 1 / 2$, whereas only background level activity was observed in cells with the empty vector control (Figure 2D). This result is consistent with our findings from yeast growth tests and aconitase activity assays (Figure 2A-C). Overall, our yeast results consistently supported the hypothesis that FPN3 is a mitochondrial iron exporter and is functionally analogous to Mmt1/2.

\section{FPN3 is expressed in the shoots, roots, flowers, and siliques, and is iron-regulated in roots}

To detect the expression of $F P N 3$ at the tissue level, we fused the promoter region of $F P N 3$ to the $\beta$-glucuronidase (GUS) reporter gene (FPN3p-GUS), transformed the construct into wild type Arabidopsis, and conducted GUS histochemical staining. FPN3p-GUS was expressed in shoots and roots of seedlings from an early stage, and in floral organs and siliques (Figure 3A-F), which was in agreement with FPN3 expression data reported in multiple transcriptomics studies (Schmid et al., 2005; Zimmermann et al., 2004; Dinneny et al., 2008; Winter et al., 2007). 
To determine whether FPN3 expression is regulated by iron, we performed GUS staining with FPN3p-GUS plants grown under iron-deficient or iron-sufficient conditions. We detected stronger staining in roots of seedlings from iron-deficient medium than in roots from ironsufficient medium, whereas FPN3p-GUS staining in the shoots was prominent under both conditions (Figure 3I-K). FPN3p-GUS expression in 1-day old seedlings exhibited similar patterns, with more intense staining in the roots of plants grown under iron-deficient conditions compared to plants from iron-sufficient conditions (Figure 3G, H). Staining in the shoots was similar under both iron conditions. This observation corroborates our RT-qPCR results in which FPN3 transcript level was approximately 4.5-fold higher in iron-deficient roots (Figure 3L), and is consistent with transcriptomics studies that reported higher steady state levels of FPN3 transcripts in iron-deficient roots (Mai et al., 2016; Dinneny et al., 2008; Yang et al., 2010; Park et al., 2019; Khan et al., 2018; Buckhout et al., 2009). Meanwhile, the constitutive expression of FPN3 was detected in the shoots regardless of the iron status of the plant (Figure 3I, L).

\section{FPN3 is iron-regulated primarily by local signals in roots}

Studies with multiple plant species have revealed that two types of iron-deficiency signals regulate the iron-deficiency response in the roots: the local signal determined by the iron level in the rhizosphere, and the systemic, long distance signal based on the iron status of the shoots (Vert et al., 2003; Grusak and Pezeshgi, 1996; García et al., 2013; Bienfait et al., 1987).

The differential level of FPN3 transcripts in roots and shoots prompted us to test if the irondependent FPN3 accumulation in roots is regulated by a local or long-distance signal. Thus, we generated two lateral roots, or split roots, and each root was subjected to different iron growth conditions. The iron growth conditions for our split-root experiments were optimized based on the method by Kumar et al. (2017). Plants were grown under iron-deficient or iron-sufficient 
conditions for 3 days prior to the split media experiment and RNA was extracted from each side of the split root after 0,6 , or 12 hours after transfer (Figure 4A) for RT-qPCR. If FPN3 level in the roots is regulated by a long-distance signal from the shoots, then FPN3 expression is expected to be higher in both sides of the split roots of plants that were initially grown in iron deficient medium, regardless of the iron conditions for each side of the split roots. Our results revealed that, regardless of the initial growth conditions, significantly more FPN3 transcripts accumulated in the split root subjected to iron-deficiency compared to its counterpart grown in iron sufficient medium (Figure 4B). This suggests that the increased level of FPN3 transcripts in iron deficient roots is primarily driven by the local iron conditions (Figure 4B). In the split root transferred from iron sufficient conditions to iron deficient conditions, we observed the greatest increase in FPN3 transcript levels (Figure 4B). Meanwhile, between the split roots pre-treated under iron deficiency, only the split root subjected to an additional iron deficiency exhibited higher levels of FPN3 over the time course tested. However, in the split-root transferred from iron sufficient to sufficient conditions, it was noted that there was a 2-fold increase in FPN3 transcript level (Figure 4B). This observation suggests that a systemic signal from the other split root, experiencing iron deficiency, might be involved, suggesting a possible contribution of the shoot-to-root signaling. Overall, our split-root results suggest that FPN3 expression in roots is regulated by the local iron status in the roots, but some systemic signaling is involved as well.

\section{Growth of $f p n 3$ mutants is reduced under iron deficiency}

We then conducted phenotypic analyses with $f p n 3$ plants to understand the function of FPN3 in iron homeostasis. Based on the increased FPN3 expression in roots under iron deficiency (Figure 3I-L), we hypothesized that fpn3 mutants might have less iron available for growth or development. To test this idea, we germinated two T-DNA insertion lines, $f p n 3-1$ and $f p n 3-2$, 
which had significantly reduced levels of FPN3 transcripts (Supplementary Figure 3) in alkaline soil, in which iron availability is drastically reduced. We observed that $f p n 3$ single mutants were smaller than wild type in alkaline soil, but this phenotype was recovered when watered with soluble iron (Figure 5A, 5B). As an alternative approach to verify the low iron growth phenotype, we quantified the root lengths and shoot fresh weights of the fpn 3 and wild type seedlings germinated in iron sufficient medium (Supplementary Figure 3C) or in medium without iron (Supplementary Figure 3D). Under iron deficient conditions, fpn3 mutant seedlings exhibited decreased shoot fresh weights (Supplementary Figure 3D). The growth defect of fpn3 on iron-deficient medium and alkaline soil consistently showed that FPN3 is necessary for optimal growth under iron limiting conditions.

\section{Iron content is reduced in fpn 3 mutant leaves}

To test if iron content of fpn 3 mutant leaves is affected, we conducted bulk elemental analysis of shoot and root tissues from plants grown under iron deficient and sufficient conditions using inductively coupled plasma mass spectrometry (ICP-MS). ICP-MS results revealed that iron content of fpn 3 mutant shoot was significantly lower than that of wild type under both iron sufficient and deficient conditions (Figure 6A). In roots, iron content was not statistically different between wild type and fpn3 mutants under iron sufficient conditions while iron content was significantly lower in fpn3 mutant roots than in wild type roots under iron deficiency (Figure 6B). We then performed synchrotron $x$-ray fluorescence imaging (SXRF) to examine the amount and distribution of iron in leaves of $f p n 3$ and wild type. The overall distribution of iron was not different among genotypes (Figure 6C). The elemental map of manganese and zinc were similar compared to wild type (Supplementary Figure 4). These results suggested that the function of FPN3 is necessary to properly maintain iron levels in the leaves regardless of iron conditions and 
in the roots under iron deficiency. We note that our ICP-MS data correlates with the expression profile of FPN3 in roots and shoots under iron sufficient and deficient conditions (Figure 3L).

\section{FPN3-GFP is dual-targeted to the mitochondria and plastids in Arabidopsis}

To determine the subcellular localization of FPN3, we generated transgenic Arabidopsis plants that stably express $35 S p-F P N 3-G F P$ and examined the subcellular localization of FPN3-GFP in roots after staining with MitoTracker Red, a mitochondrial dye. In transgenic roots, FPN3-GFP signal co-localized with the fluorescence of MitoTracker Red (Figure 7A-C). Based on quantification, $83 \%$ of the GFP signal co-localized with the mitochondrial staining. The FPN3GFP signal that did not overlap with the mitochondrial staining (Figure 7C) suggested that FPN3-GPF might be dual-targeted. To confirm the localization of FPN3-GFP in chloroplasts, we also generated transgenic Arabidopsis plants stably expressing both 35Sp-FPN3-GFP and 35Sp$p t-R F P$, a plastid marker with the transit peptide of rubisco small subunit from tobacco fused to RFP (Nelson et al., 2007). We observed 72\% co-localization of FPN3-GFP with the plastid marker (Figure 7D-F). Overall, our localization results from both stable transgenic lines confirm the plastid localization of FPN3-GFP previously reported by Conte et al. (2009) and provide further evidence that FPN3 is dual-targeted.

\section{Mitochondria and chloroplasts of $f p n 3$ accumulate iron}

Based on the subcellular localization of FPN3 in the mitochondria and chloroplasts (Figure 7AF) and our yeast results that suggested FPN3 is exporting iron from these organelles (Figure 2), we predicted that iron would accumulate in the mitochondria and chloroplasts of $f p n 3$. Thus, we isolated these organelles from $f p n 3$ and wild type shoots. To examine the purity of our samples, Western blots were done with the chloroplasts or mitochondrial samples using antibodies against 
cytosolic or organellar markers (Supplementary Figures 5, 6). We then quantified metal concentrations using ICP-MS. As predicted, chloroplasts and mitochondria from both fpn3-1 and fpn3-2 contained significantly more iron compared to their counterparts in wild type (Figure $7 \mathrm{G}$, 7H). The accumulation of iron in fpn 3 mutant chloroplasts and mitochondria provides strong evidence that supports the role of FPN3 in exporting iron from the mitochondria and chloroplast.

\section{The expression of iron responsive genes is altered in the $f p n 3$ mutants}

Trafficking of iron between subcellular compartments is crucial for cellular iron homeostasis. Because mitochondria and chloroplasts prepared from leaves of $f p n 3$ mutants accumulate more iron, while leaves of the mutant accumulate less iron compared to wild type, we predicted that transcript levels of organellar iron responsive genes would be affected in $f p n 3$ mutant lines. We first analyzed the expression of vegetative ferritin genes, FERRITIN1 (FER1), FER3, and FER4, which transcripts accumulate under iron sufficiency and decrease when iron is low (Petit et al., 2001; Arnaud et al., 2006). We found that transcript levels of FER1 and FER3, which encode plastid-localized ferritins, were significantly lower in iron-sufficient shoots of $f p n 3$ compared to wild type (Figure 8), which is consistent with our results showing less iron accumulation in fpn3 shoots (Figure 6A). In contrast, the expression of FER4, a dual-targeted ferritin that localizes to mitochondria and plastids, was approximately 2 and 2.9-fold higher in fpn3-1 and fpn3-2, respectively, compared to wild type (Figure 8).

FRO3 encodes a mitochondrial ferric chelate reductase and has been used as a marker for iron deficiency (Mukherjee et al., 2006). FRO3 expression was about 46\% and 31\% higher in irondeficient shoots of $f p n 3-1$ and fpn3-2, respectively, compared to wild type (Figure 8). In addition, FRO3 expression was significantly higher in $f p n 3$ shoots than in wild type under iron-sufficient 
conditions (Figure 8). Along with the expression profiles of FER1 and FER3, elevated FRO3 expression levels in fpn 3 shoots is consistent with less cytosolic iron (Figure 6). Expression of another mitochondrial ferric chelate reductase gene, FRO8, was $\sim 30 \%$ higher in iron-deficient $f p n 3$ shoots than in iron-sufficient $f p n 3$ shoots, and was increased 2-fold in $f p n 3$ shoots compared to wild type shoots under iron-deficient conditions (Figure 8). In contrast to the mitochondrial ferric chelate reductase genes, expression of FRO7, which encodes the chloroplast ferric chelate reductase, was not significantly different between the $f p n 3$ mutants and wild type; FRO7 transcript levels were increased in iron deficiency, but similar across all three genotypes (Figure 8). We noted that the expression of YSL4 and YSL6, which have been reported to encode effluxors of iron-NA complexes from the chloroplasts (Divol et al., 2013) or vacuoles (Jaquinod et al., 2007; Conte et al., 2013), was significantly increased in fpn3 mutant shoots as compared to wild type (Figure 8). NRAMP4 encodes a vacuolar iron effluxer and is induced by iron deficiency (Lanquar et al., 2005). Compared to wild type, NRAMP4 expression was significantly higher in iron-deficient shoots of fpn3 (Figure 8). This observation is in accordance with the expression profiles of FER1, FER3 and FRO3 in shoots, which suggested that fpn 3 shoots have less iron than wild type shoots, and our elemental analysis data, which showed that fpn3 shoots contain less iron than wild type shoots (Figure 6).

\section{fpn3 mitochondria exhibit abnormal morphology under iron deficient conditions}

Abnormal chloroplast ultrastructure has been observed in mutants defective in iron metabolism of multiple plants species grown under iron deficiency (Bogorad et al., 1959; Duy et al., 2007; Platt-Aloia et al., 1983; Stocking, 1975; Vigani et al., 2015). In addition to chloroplasts, the ultrastructure of mitochondria has also been shown to be affected by iron deficiency in plants (Pascal and Douce, 1993; Vigani et al., 2015). Therefore, we examined the ultrastructure of 
chloroplasts and mitochondria in fpn3 and wild type leaves using transmission electron microscopy (TEM). Our TEM images revealed that mitochondria of iron-deficient fpn3 mutants were enlarged and the outer and inner membranes appeared deformed (Figure 9C), but such phenotypes were not detected under iron sufficient conditions (Figure 9A). By comparing the quantified area of mitochondria, we determined that $f p n 3$ mitochondria were significantly larger than wild type mitochondria under iron deficient conditions (Figure 9D), but not under iron sufficient conditions (Figure 9B). Overall, the iron-dependent mitochondrial ultrastructure phenotype (Figure 9) strongly suggests that FPN3 function is critical for mitochondria under iron deficient conditions.

\section{DISCUSSION}

\section{FPN3 is a dual-targeted mitochondrial/chloroplast iron exporter}

FPNs have been extensively studied in vertebrate species, in which the only FPN is localized to the plasma-membrane and exports iron from the cell into the plasma (Drakesmith et al., 2015). In many plant species, multiple FPN/IREG paralogs are present and localize to different subcellular compartments. FPN1/IREG1 in Arabidopsis thaliana (Morrissey et al., 2009) and the nodule-specific FPN2 in Medicago truncatula (Escudero et al., 2020) are targeted to the plasma membrane, whereas the Arabidopsis FPN2/IREG2 (Morrissey et al., 2009; Schaaf et al., 2006), IREG1 of the nickel hyperaccumulator Psychotria gabriellae (Merlot et al., 2014), and buckwheat IREG (Yokosho et al., 2016) were identified on the tonoplast. Although MAR1 (alias FPN3/IREG3) was reported to be targeted to the chloroplasts (Conte et al., 2009), our study shows that is dual-targeted to mitochondria and chloroplasts (Figure 7A-F). 
This study suggests that FPN3 is exporting iron from the mitochondria and chloroplasts. FPN family members identified to date transport iron from the cytoplasm. As metal transporters of the same family generally retain the direction of transport in respect to the cytoplasm, an organellar FPN is likely to transport iron into the organellar lumen. However, the endosymbiotic origin of mitochondria and chloroplasts (Roger et al., 2017; Zimorski et al., 2014; Yoon et al., 2004) suggests that FPN3 might efflux iron from its ancestral cytoplasm, i.e., from the organellar lumen into the eukaryotic cytoplasm. Under the hypothesis that FPN3 is of bacterial origin, its directionality is consistent with iron export from its ancestral cytoplasm. Based on the subcellular localization of FPN3 (Figure 7A-F) and the endosymbiotic origin of mitochondria and chloroplasts (Roger et al., 2017; Zimorski et al., 2014; Raven and Allen, 2003; Rockwell et $a l .$, 2014), it is reasonable to speculate that FPN3 diverged from a bacterial ancestor. Prokaryotic ferroportins have not been extensively studied, but a bacterial FPN from Bdellovibrio bacteriovorus has been reported (Bonaccorsi di Patti et al., 2015; Taniguchi et al., 2015).

\section{The physiological role of FPN3}

FPN3 was previously reported as MAR1 and proposed to opportunistically transport aminoglycoside class antibiotics (Conte et al., 2009; Conte and Lloyd, 2010). In this report, we add new information that is critical to understanding the physiological role of FPN3/MAR1. First, our results show that FPN3 is dual-targeted to the mitochondria and chloroplasts and affects mitochondrial morphology under iron deficiency (Figures 7, 9). Conte et al (2009) reported that MAR1 was targeted to chloroplasts. Here, we observed that FPN3-GFP is colocalized with mitochondrial and plastid markers (Figure 7A-F). Furthermore, we detected iron accumulation in both mitochondria and chloroplasts of $f p n 3$ (Figure 7G, H) and observed abnormal mitochondrial morphology in iron deficient fpn3 mutant leaves (Figure 9). Our TEM 
results indicate that FPN3 plays the essential role in maintaining iron homeostasis in the mitochondria. It is also possible that the chloroplasts may be better equipped with mechanisms that compensate for defective FPN3 function under iron deficient conditions. For example, given the proposed function of YSL4/6 in chloroplasts (Divol et al., 2013), we speculate that YSL4/6 might be compensating for the loss of FPN3 in plastids. Alternatively, based on their proposed role in the vacuole (Conte et al., 2013), the increased YSL4/6 transcript levels in fpn3 is consistent with the higher level of NATURAL RESISTANCE-ASSOCIATED MACROPHAGE PROTEIN 4 (NRAMP4) expression observed in fpn3 (Figure 8). In addition, three ferritin genes are expressed in chloroplasts (FER1, FER3 and FER4), while FER4 is the only mitochondrial ferritin.

Second, we show that FPN3 exports iron from mitochondria and chloroplasts and most likely transports ferrous iron ions. Conte et al (2009) speculated that MAR1 may be importing the polyamine iron chelator, nicotianamine (NA), based on its structural similarity to aminoglycoside class antibiotics. However, the presence of the highly conserved iron ion binding residues in FPN3/IREG3 (Figure 1) and the iron toxicity phenotypes of yeast cells expressing FPN3 that are analogous to yeast cells expressing MMT1/2 (Figure 2) strongly support the idea that ferrous iron ions are FPN3/IREG3 substrates. Furthermore, our results support the role of FPN3/IREG3 as an iron exporter of mitochondria and chloroplasts, based on the yeast data (Figure 2) and iron accumulation quantified by ICP-MS with isolated chloroplasts and mitochondria (Figure 7G, H). It is noteworthy that in a recent study, BnMAR1, the FPN3/IREG3 ortholog in Brassica napus, was speculated to be involved in iron release from the plastids based on the correlation between its gene expression and chloroplast iron content (Pham et al., 2020). Although the mechanism of transport via FPN has not been fully understood, structural and 
biochemical studies indicated that the bacterial FPN3 ortholog, BdFPN, transports iron and other divalent cations along their concentration gradient in a uniporter-like manner (Taniguchi et al., 2015). We noted that the content of other essential metals, such as manganese, zinc, and copper, was also affected in fpn3 chloroplasts and mitochondria (Supplementary Figure 7), which suggests that FPN3 might transport other metals in addition to iron. In particular, zinc accumulation was significantly higher in both mitochondria and chloroplasts of $f p n 3$ than in wild type (Supplementary Figure 7) and SXRF indicated that fpn3 leaves contained less zinc than wild type leaves (Supplementary Figure 4). While manganese and copper accumulation in fpn3 mitochondria and chloroplasts was also significantly different from that of wild type, this may reflect secondary effects because the pattern of changes was not consistent between the two organelles (Supplementary Figure 7). Further studies will be necessary to test if other metals could also be transported by FPN3.

Third, we show that FPN3 is constitutively expressed regardless of the iron conditions in the shoots, whereas its transcript levels are increased by iron deficiency in the roots. We note that our data contradicts the results of Conte et al (2009), who reported the down-regulation of MARI expression in low iron conditions by RT-qPCR with whole seedlings. However, multiple transcriptomics datasets corroborate our results and show that FPN3/IREG3 transcript level is higher in wild type roots under iron deficiency (Mai et al., 2016; Dinneny et al., 2008; Yang et al., 2010; Park et al., 2019; Khan et al., 2018; Buckhout et al., 2009) but is not iron-regulated in shoots (Khan et al., 2018; Rodríguez-Celma et al., 2013). The differential expression of FPN3 in roots and shoots under iron-deficient or iron-sufficient conditions (Figure 3G-L) implies that FPN3 might contribute to different physiological roles in roots and shoots. Genes involved in iron acquisition or mobilization are generally induced under iron deficiency (Connolly et al., 
2002; Varotto et al., 2002; Vert et al., 2002; Lanquar et al., 2005; Thomine et al., 2003), whereas steady-state transcript levels of multiple metal homeostasis genes that load iron into subcellular organelles remain unaffected by the availability of metals in the environment (Kim et al., 2006; Zhang et al., 2012; Desbrosses-Fonrouge et al., 2005; Klaumann et al., 2011). Our split root data revealed that the transcript level of FPN3 in roots is regulated in response to the local availability of iron, but also indicated that systemic signals also contribute to FPN3 regulation (Figure 4). This suggests that FPN3 is releasing iron from chloroplasts/plastids and mitochondria in the roots when iron deficiency in the rhizosphere is sensed, while responding to iron levels in the shoots. We speculate that FPN3 may be releasing iron into the root cytoplasm so that it can be reallocated within the cell to help cope with iron deficiency and to adjust metabolic needs under iron deficiency. As a response to iron deficiency, metabolic pathways in plants, including those that occur in chloroplasts and mitochondria, are coordinately remodeled in both roots and shoots (López-Millán et al., 2013; Thimm et al., 2001; Rodríguez-Celma et al., 2013). In the shoots, FPN3 is constitutively expressed regardless of the plant iron status (Figure 3G-L) and throughout development according to transcriptomic datasets (Winter et al., 2007). FPN3 might be releasing iron to protect from iron-induced oxidative stress in the shoots. It has been proposed that plant ferritins play a major role in preventing iron-induced oxidative stress in plants (Ravet et al., 2009). FER4 encodes a dual-targeted ferritin that localizes to mitochondria and plastids. FER4 is the only mitochondrial ferritin, as mitochondria of fer4 cells are devoid of ferritins (Tarantino, Santo, et al., 2010). Thus, the pronounced increase in FER4 transcripts is likely caused by fpn3-1 and fpn3-2 mutant plants accumulating more iron in the mitochondria and chloroplasts and, in response, higher level of FER4 transcripts to prevent iron-induced oxidative damage. 
Although FPN3 does not appear to be iron-regulated at the transcriptional level in the shoots, the abnormal mitochondria morphology detected in iron deficient fpn3 leaves suggest a critical role for FPN3 in the mitochondria under iron deficiency (Figure 9). Because FRO8 is not regulated by the iron status of wild type plants (Figure 8;(Mukherjee et al., 2006), the higher level of FRO8 in iron-deficient fpn3 shoots (Figure 8) may also imply a dysregulation of iron-dependent mitochondrial function. While the mechanism behind the enlarged mitochondria of iron deficient fpn3 shoots remains to be understood, it is noteworthy that impaired ion homeostasis is known to affect mitochondrial volume (Szabo and Zoratti, 2014; Teardo et al., 2015). Swollen mitochondria have been observed upon anaerobic treatment, decreased metabolism, or oxidative stress (Vartapetian et al., 2003; Yoshinaga et al., 2005; Lee et al., 2002). We postulate that oxidative stress or metabolic constraints caused by dysregulated iron homeostasis in iron deficient $f p n 3$ mitochondria might have resulted in the compromised mitochondrial ultrastructure. We further speculate that the reduced copper level in fpn3 mitochondria (Supplementary Figure 7B) may indicate that fpn3 mitochondria are more susceptible to oxidative stress than $f p n 3$ chloroplasts. A recent study has shown that mitochondria accumulates oxygen under low copper due to impaired cytochrome c function (Ishka and Vatamaniuk, 2020).

\section{Implications of a mitochondrial iron exporter}

Mitochondrial metabolism was found to be less affected than chloroplast metabolism under mild iron deficiency (Hantzis et al., 2017). However, it is noteworthy that improper FPN3 function appears to have a greater impact on the mitochondria than chloroplasts (Figure 9). The mitochondrial phenotype of $f p n 3$ signifies the importance of iron export from the mitochondria under iron deficient conditions. It has been well-established that chloroplasts are the most ironrich organelle in plant cells and vacuoles serve as the major iron storage compartment, and 
several studies have provided evidence that mitochondria can contribute as an additional intracellular iron reservoir. In yeast $\Delta c c c 1$ mutant cells that lack the vacuolar iron importer Ccc1, overexpressing MRS3 and MRS4, which encode mitochondrial iron importers of the mitoferrin family, suppressed iron toxicity (Lin et al., 2011). Furthermore, MMT1 and MMT2, which belong to the cation diffusion facilitator (CDF) family, export mitochondrial iron and overexpression of $M M T 1 / 2$ results in multiple phenotypes that support the idea that mitochondria could function as an iron storage organelle (Li et al., 2014). It is interesting to note that mitochondrial iron import mediated by mitoferrins is common across multiple organisms, such as in yeast, mammals, worm, fruit fly, rice, and Arabidopsis (Paradkar et al., 2009; Shaw et al., 2006; Metzendorf et al., 2009; Ren et al., 2012; Bashir et al., 2011; Mühlenhoff et al., 2003; Jain et al., 2019). Unlike mitochondrial iron import, we speculate that mechanisms to export mitochondrial iron are more diverse. FPNs do not exist in yeast and mitochondrial FPNs have not been found in vertebrates or invertebrates, to our knowledge. In mammalian mitochondria, an ATP-binding cassette $(\mathrm{ABC})$ transporter, $\mathrm{ABCB} 8$, is involved in exporting iron (Ichikawa et al., 2012). The presence of a mitochondrial iron exporter is necessary, but not sufficient to suggest that mitochondria might function as a subcellular iron store. Nevertheless, our work shows that FPN3 is regulating mitochondrial iron and implies a potential role of mitochondria as an iron reservoir. It is possible that fpn 3 mutants might be increasing FER4 to protect from iron toxicity due to the lack of an iron exporter. Furthermore, the higher expression of FER4 in fpn 3 than in wild type (Figure 8) is also consistent with the view that mitochondria could store iron. We postulate that mitochondrial ferritin may play a more dynamic function to assist iron storage in addition to protecting mitochondria from iron-induced oxidative damage. 
In conclusion, our work has advanced our understanding of the physiological roles of FPN3 by providing evidence that FPN3 is an iron exporter dual-targeted to mitochondria and chloroplasts. Our TEM results imply that FPN3 function is more crucial for mitochondria, but further studies are necessary to elucidate the mechanisms underlying the phenotypes observed and the potential effect of FPN3 on mitochondrial and chloroplast metabolism and oxidative stress. Future work should also address potential crosstalk among chloroplasts, mitochondria, and vacuoles to comprehensively understand organellar iron trafficking and homeostasis in plant cells.

\section{MATERIALS AND METHODS}

\section{Yeast high iron growth assays}

For growth tests in high iron media, $p R S 315-F P N 3, p R S 315-M M T 1 / 2$, or $p R S 315$ vector alone was transformed into $\Delta c c c 1 \Delta m m t 1 / 2$ cells. For spot assays, serial dilutions of overnight yeast cultures were spotted onto SD-Leu plates with appropriate auxotrophic supplements containing 2 $\mathrm{mM}$ iron (2 $\mathrm{mM}$ ferrous ammonium sulfate hexahydrate). The negative control plates contained media with no added iron. Cells growth at $30^{\circ} \mathrm{C}$ was monitored over 2-5 days. For growth assays in liquid culture, overnight yeast cultures were inoculated to a starting $\mathrm{OD}_{600}$ of 0.05 into SDLeu with or without $2 \mathrm{mM}$ iron supplementation and grown in a shaker incubator at $30^{\circ} \mathrm{C}$, and cell density was measured at $\mathrm{OD}_{600}$ at the indicated time points.

\section{Aconitase assay}

Wild type DY150 cells were transformed with FPN3, MMT1/2, or $p R S 315$ vector alone. These transformants were inoculated in appropriate selective media (SD -Leu), grown overnight at $30^{\circ} \mathrm{C}$, and sub-cultured in SD-Leu supplemented with $250 \mu \mathrm{M} \mathrm{FeSO}_{4}$ until the cells reached mid- 
$\log$ phase. The subcultures were harvested and washed with cold phosphate-buffered saline (PBS), and protein lysate was prepared using PBS with protease inhibitor (Pierce) via homogenization with glass beads for 10 minutes at $4^{\circ} \mathrm{C}$. Samples used in the aconitase assay were normalized with total protein concentration. Aconitase activity was assayed and quantified using the BioAssay Systems EnzyChrom Aconitase Assay Kit, following manufacturer's instructions.

\section{c-GDO assay}

Wild type DY150 cells were co-transformed with FPN3, MMT1/2, or $p R S 315$ and the $c-G D O$ plasmid, and grown as described above for the aconitase assay. Cell lysates were prepared with these transformants by glass bead homogenization, and GDO activity was assayed as described by $\mathrm{Li}$ et al (2014). The assay reaction consisted of $20 \mathrm{mM}$ Tris- $\mathrm{HCl}(\mathrm{pH} 8.0)$ and $0.1 \mathrm{mM} 2,3-$ dihydroxy-benzoic acid (gentisic acid) as the substrate. The enzyme activity was determined based on the absorbance measured at $340 \mathrm{~nm}$ using an extinction coefficient of $10.2 \mathrm{~cm}^{-1} \mathrm{~mm}^{-1}$.

\section{Plant materials and growth conditions}

Two independent alleles of fpn3 T-DNA insertion lines, fpn3-1 (SALK_034189) and fpn3-2

(SALK_009286), were obtained from the Salk collection (Alonso et al., 2003). RT-qPCR verified that FPN3 expression was drastically reduced in these lines (Supplementary Figure 3). Arabidopsis plants were grown at $22{ }^{\circ} \mathrm{C}$ under a 16/8-hour light/dark cycle in the Conviron A1000 growth chamber. 


\section{Plant growth test under iron-deficient conditions}

For alkaline soil tests, seedlings were germinated and grown in normal soil ( $\mathrm{pH} \sim 5.8)$, alkaline soil ( $\mathrm{pH} \sim 8)$, or alkaline soil watered with soluble iron (0.5 mM Fe-EDDHA). Alkaline soil was prepared by adding approximately $1.3 \mathrm{~g}$ of calcium oxide per liter of soil solution (Kim et al., 2006). For growth tests on plates, seedlings were germinated vertically for 12 days on either iron-deficient media (Murashige and Skoog (MS) without iron (Caisson MSP33) supplemented with $300 \mu \mathrm{M}$ ferrozine) or iron-sufficient media (MS with $100 \mu \mathrm{M}$ FeNa-EDTA (Caisson MSP34). Seedlings were photographed, and ImageJ was used to quantify root lengths. Shoots were separated and weighed on the MT5 Analytical Microbalance (Mettler Toledo).

\section{Generation of FPN3p-GUS transgenic lines and histochemical staining}

A fragment containing 890 bp of upstream sequence and the FPN3 start codon was amplified from a BAC clone, F2P16 (GenBank AF007270), obtained from the Arabidopsis Biological Resource Center (ABRC) using primers 5'-cacccactttctcttttgttagattctagttg-3' and 5'-cattctataaattgattctcctcttctcc-3' and cloned into pENTR/D-TOPO (Invitrogen). The construct was cloned into pGWB533 (Nakagawa et al., 2007) using Gateway LR Clonase II (Invitrogen). The resulting FPN3p-GUS construct was moved into Agrobacterium tumefaciens strain LBA4404 and transformed into wild type Col 0 plants using the floral dip method (Clough and Bent, 1998). pGWB533 was from Tsuyoshi Nakagawa (Addgene plasmid \#74872; RRID:

Addgene_74872). For histochemical staining, seedlings or tissues of FPN3p-GUS plants were fixed and incubated with 5-bromo-4-chloro-3-indolyl $\beta$-D-glucuronide as described by (Jefferson et al., 1987). At least five seedlings from three independent T2 or T3 lines were examined, and images were obtained using the LeicaM165 FC dissecting scope and Leica LAS EZ 3.4.0 software. 


\section{Split root assays}

For split-root samples for RT-qPCR, plants were prepared following the protocol described by Kumar et al. (2017) with slight modifications. Wild type plants were grown for 5 days on ironsufficient medium, cut at the roots and grown for 7 days to induce split roots, and transferred to iron-deficient or iron-sufficient medium for 3 days. The plants were then washed with $0.1 \mathrm{M}$ citrate buffer and moved to split plates, in which one of the split roots was placed on irondeficient medium and the other root on iron-sufficient medium. Plants were kept on split plates for 0,6 and 12 hours before harvesting roots for RNA preparation.

\section{Generation of FPN3-GFP transgenic lines}

The coding region of FPN3 was cloned from a BAC clone, F2P16 (GenBank AF007270), obtained from the Arabidopsis Biological Resource Center (ABRC) using primers 5'caccatggttgtttcaatggctttgg-3' and 5' -atttgagagagggtcgaaggag-3' and cloned into pENTR/DTOPO (Invitrogen). The final construct, 35Sp-FPN3-GFP, was cloned into pGWB505 (Nakagawa et al., 2007) using Gateway LR Clonase II (Invitrogen). 35Sp-FPN3-GFP and 35Sppt-RFP were co-transformed into Agrobacterium tumefaciens strain LBA4404, and then into wild type Col 0 using the floral dip method (Clough and Bent, 1998). pGWB505 was a gift from Tsuyoshi Nakagawa (Addgene plasmid \#74847; RRID: Addgene_74847) and 35Sp-pt-rb (Nelson et al., 2007) was obtained from ABRC (stock \#CD3-1000).

\section{Confocal microscopy}

Arabidopsis roots stably expressing 35Sp-FPN3-GFP were imaged with the Nikon A1 Spectral Detector Confocal with FLIM Module at the Light Microscopy Core, Institute for Applied Life Science at University of Massachusetts, Amherst. For mitochondrial co-localization roots were stained with MitoTracker Red FM (Invitrogen) 28 label the mitochondria prior to imaging, by 
incubating roots in a final concentration of $1 \mu \mathrm{M}$ MitoTracker Red FM for 30 minutes. Z-stack images were taken with FITC and TRITC channels. Images were collected and processed with NIS-Elements software.

\section{Chloroplast isolation}

Chloroplast isolation was conducted following (Smith et al., 2003). Arabidopsis plants were grown for 4 weeks on soil. Roughly $10 \mathrm{~g}$ of shoot tissue was harvested, rinsed in digestion buffer (20 mM MES-KOH, pH 5.2, $400 \mathrm{mM}$ sorbitol, $0.5 \mathrm{mM} \mathrm{CaCl}_{2}$ ), and incubated in digestion enzyme solution (10 ml digestion buffer, $0.04 \mathrm{~g}$ Macerozyme R-10 (bioWORLD), $0.2 \mathrm{~g}$ Cellulase (bioWORLD) for $3.5 \mathrm{~h}$ under light. During digestion, a 40\%:85\% AT Percoll step gradient was prepared on ice. Upon completion of digestion, the solution was filtered through cheesecloth to harvest protoplasts. To isolate protoplasts, the filtered solution was suspended in digestion buffer and centrifuged at $100 \mathrm{~g}$ for $5 \mathrm{~min}$ at $4^{\circ} \mathrm{C}$, the supernatant was discarded, then the isolated protoplasts were resuspended in protoplast resuspension buffer (20 mM MES-KOH, pH 6.0, $400 \mathrm{mM}$ sorbitol, $0.5 \mathrm{mM} \mathrm{CaCl}_{2}$ ) before centrifuging at $100 \mathrm{~g}$ for $2 \mathrm{~min}$ at $4^{\circ} \mathrm{C}$ and removing supernatant. Next, protoplasts were resuspended in protoplast breakage buffer (20 mM tricine-KOH, pH 8.4, 300 mM sorbitol, 5 mM EDTA, 5 mM EGTA, 10 mM NaHCO $3,0.05$ g BSA added per $50 \mathrm{~mL}$ of solution before use) and immediately filtered through a protoplast rupturing device (a syringe tube with the end cut off, with a $20 \mu \mathrm{m}$ and $10 \mu \mathrm{m}$ mesh, respectively, attached via electrical tape). Next, the broken chloroplasts were layered onto the 40\%:85\% AT Percoll step gradient and centrifuged at $2500 \mathrm{~g}$ for $10 \mathrm{~min}$ at $4^{\circ} \mathrm{C}$, with the brake off. A green band was visualized at the $40 \% 85 \%$ AT Percoll interface and harvested. The resulting solution was diluted in HEPES-sorbitol buffer, pH 8.0 (50 mM HEPES-KOH, pH 8.0, 
$330 \mathrm{mM}$ sorbitol) and centrifuged at $700 \mathrm{~g}$ for $5 \mathrm{~min}$ at $4^{\circ} \mathrm{C}$. After removing the supernatant, resulting chloroplasts were resuspended in HEPES-sorbitol buffer, $\mathrm{pH}$ 8.0.

Chlorophyll content (chlorophyll $a$ and $b$ ) was quantified with the NanoDrop One Spectrophotometer (ThermoFisher) following manufacturer's instructions. The following equations were used to quantify chlorophyll $a$ and chlorophyll $b$ content, respectively: $C_{\mathrm{a}}=$ $14.85 A^{666}-5.14 A^{650} ; C_{\mathrm{b}}=25.48 A^{650}-7.36 A^{666}$ (Porra et al., 1989; Barnes et al., 1992). Purity was confirmed via Western blots with organellar markers.

\section{Mitochondrial isolation}

Mitochondria were isolated from four-week-old Arabidopsis leaves using a protocol adapted from (Keech et al., 2005). Prior to the isolation, 30mL of continuous 50\% Percoll gradient (50\% (v/v) Percoll, 0.3M sucrose, $10 \mathrm{mM}$ TES, $1 \mathrm{mM}$ EDTA, $10 \mathrm{mM} \mathrm{KH}_{2} \mathrm{PO}_{4}, 1 \mathrm{mM}$ glycine, $\mathrm{pH}$ adjusted to 7.5 with $\mathrm{KOH}$ ) was centrifuged at $39000 \mathrm{~g}$ for $40 \mathrm{~min}$ and kept at $4^{\circ} \mathrm{C}$. Roughly 40 $50 \mathrm{~g}$ of tissue was homogenized in pre-chilled grinding buffer B (0.3M sucrose, $60 \mathrm{~m}$ TES, $2 \mathrm{mM}$ EDTA, 10mM KH $2 \mathrm{PO}_{4}, 25 \mathrm{mM}$ tetrasodium pyrophosphate, $1 \mathrm{mM}$ glycine, 1\% (w/v) polyvinylpyrrolidone-40, 1\%(w/v) defatted bovine serum albumin (BSA), with $50 \mathrm{mM}$ sodium ascorbate and $20 \mathrm{mM}$ cysteine added and readjustment of the $\mathrm{pH}$ to 8.0 with $\mathrm{KOH}$ just prior to grinding) using a hand blender, filtered through a $20-\mu \mathrm{m}$ nylon mesh, and centrifuged at $2500 \mathrm{~g}$ for 5 minutes to remove intact chloroplasts and thylakoid membranes. The supernatant was centrifuged at $15000 \mathrm{~g}$ for 15 minutes. The pellet obtained was resuspended in wash buffer B (0.3M sucrose, $10 \mathrm{mM}$ TES, $10 \mathrm{mM} \mathrm{KH}_{2} \mathrm{PO}_{4}, \mathrm{pH}$ adjusted to 8.0 with $\left.\mathrm{KOH}\right)$ and gently homogenized in a chilled glass homogenizer. The resuspended pellet was then layered on top of the $50 \%$ Percoll gradient and centrifuged at $15000 \mathrm{~g}$ for 20 minutes; each $30 \mathrm{~mL}$ density gradient has a maximum load of mitochondria from $30 \mathrm{~g}$ of fresh leaf tissue. After centrifugation, the 
mitochondria formed a whitish band near the bottom of the tube. This band was aspirated and resuspended in $0.2-0.3 \mathrm{~mL}$ of wash buffer $\mathrm{B}$ to obtain a roughly 20 -fold dilution. All procedures were conducted in the cold room or at $4^{\circ} \mathrm{C}$. Mitochondrial protein was quantified using DC Protein Assay (Bio-Rad).

\section{SXRF Imaging}

The spatial distribution of iron, manganese, and zinc in hydrated leaf tissues was imaged via SXRF at the F3 station, a bending magnet beamline with multilayer monochromator, at the Cornell High Energy Synchrotron Source. Seeds were directly germinated on soil and grown at $22^{\circ} \mathrm{C}, 14 \mathrm{~h}$ light $/ 10 \mathrm{~h}$ dark photoperiod at a photo flux density of $110 \mu \mathrm{mol} / \mathrm{m}^{2} / \mathrm{sec}$. The nutrient solution contained the following components: $1.25 \mathrm{mM} \mathrm{KNO}_{3}, 0.625 \mathrm{mM} \mathrm{KH}_{2} \mathrm{PO} 4,0.5 \mathrm{mM}$ $\mathrm{MgSO}_{4}, 0.5 \mathrm{mM} \mathrm{Ca}\left(\mathrm{NO}_{3}\right)_{2}, 10 \mu \mathrm{M}$ Fe(III)HBED, $17.5 \mu \mathrm{M} \mathrm{H}_{3} \mathrm{BO}_{3}, 3.5 \mu \mathrm{M} \mathrm{MnCl}_{2}, 0.125 \mu \mathrm{M}$ $\mathrm{CuSO}_{4}, 0.25 \mu \mathrm{M} \mathrm{ZnSO}_{4}, 0.05 \mu \mathrm{M} \mathrm{Na}_{2} \mathrm{MoO}_{4}, 2.5 \mu \mathrm{M} \mathrm{NaCl}$, and $0.0025 \mu \mathrm{M} \mathrm{CoCl}_{2}$. The hydroponic solution was changed every week. Data were analyzed and processed as described in Yan et al 2017. Briefly, the first true leaves from 22 day-old plants were detached immediately prior to imaging, placed in the wet chamber made between two layers of metal-free Kapton ${ }^{\mathrm{TM}}$ film and mounted onto $35 \mathrm{~mm}$ slide mounts. The $2 \mathrm{D}$ raster maps were acquired using a focused, monochromatic incident $\mathrm{x}$-ray beam at $12.8 \mathrm{keV}$ and photon flux of approximately $3 \times 10^{10}$ photons/sec. Samples were scanned at the resolution of $30 \times 30 \mu \mathrm{m}$ and acquisition time of 0.2 sec per data point. These settings did not cause damage to plant tissues within $6-8 \mathrm{~h}$ scans required for analysis of the full set of genotypes. Element-specific x-ray fluorescence was detected using a Vortex ME-4 Silicon Drift Detector (SDD). Calibration of XRF equipment were done using a uniform thin metal film standard during each experiment. Data were processed with Praxes, a software that employs PyMca libraries in batch mode (Solé et al., 2007). 


\section{ICP-MS analysis}

Shoot iron content of Col 0, fpn3-1, and fpn3-2 was measured by ICP-MS and normalized with dry weight. Plants were grown following the same procedure as the growth test on low iron plates. Dried shoot, root, chloroplast, and mitochondrial samples of Col 0, fpn3-1, and fpn3-2 were digested with nitric acid and analyzed with the Perkin-Elmer NexION 350D ICP-MS at the Mass Spectrometry Core, Institute for Applied Life Science at University of Massachusetts, Amherst. Metal content was recorded in parts per billion (ppb) and normalized to the dry mass (mg; shoot and root tissue), chlorophyll content ( $\mu \mathrm{g}$; isolated chloroplast) or total protein ( $\mu \mathrm{g}$; isolated mitochondria) of each sample.

\section{Gene expression analyses}

Total RNA isolated with the Agilent Plant RNA Minikit from root or shoot tissue of plants grown under iron-deficient or iron-sufficient conditions were used for gene expression analyses. The quantity and purity of RNA were examined using NanoDrop One (Thermo Scientific), and the integrity of RNA was assessed by electrophoresis using a bleach gel (Aranda et al., 2012). For RT-qPCR, first strand cDNA was synthesized from total RNA using Quanta qScript, and qPCR was conducted using Bio-Rad SYBR Green Supermix in CFX-Connect Real-Time PCR Detection System (Bio-Rad). The relative transcript level of genes was calculated following the $\triangle C$ t method (Schmittgen and Livak, 2008), using ACTIN2 (ACT2) as an internal control. Primers were designed using QuantPrime (Arvidsson et al., 2008) and the sequences are listed in Supplementary Table 1. For Nanostring, a custom CodeSet that included probes specific to iron homeostasis genes of interest was designed by NanoString. Gene expression analysis was conducted with the NanoString MAX/FLEX nCounter at Dartmouth College and nCounter 
SPRINT Profiler System at the Molecular Genetics Core Facility at Boston Children’s Hospital following the manufacturer's instructions and processed using nSolver 4.0. The custom CodeSet sequences are listed in Supplementary Table 2.

\section{Transmission electron microscopy (TEM)}

Excised cotyledons of Arabidopsis seedlings treated under iron sufficient or deficient conditions were fixed by immersing them in $2.5 \%$ glutaraldehyde/ $2 \%$ paraformaldehyde in $0.1 \mathrm{M} \mathrm{Na}$ Cacodylate buffer ( $\mathrm{pH}$ 7.2) and incubating them under vacuum for $30 \mathrm{~min}$. After this primary fixation, the samples were rinsed three times in fresh fixation buffer for $10 \mathrm{~min}$. each time and were secondarily fixed with $1.0 \%$ osmium tetroxide in $\mathrm{ddH}_{2} \mathrm{O}(\mathrm{w} / \mathrm{v})$ for $1 \mathrm{hr}$ at room temperature. The samples were then rinsed again three times in $\mathrm{ddH}_{2} \mathrm{O}$ and then placed in $1 \%$ aqueous uranyl acetate $(\mathrm{w} / \mathrm{v})$ in the refrigerator overnight (tertiary fixation). After three more rinses in $\mathrm{ddH}_{2} \mathrm{O}$, the samples were then dehydrated through a graded series of ethanol $(10 \%, 30 \%, 50 \%, 75 \%$, $85 \%, 95 \%$, and $100 \%$ for 3 changes). Infiltration was accomplished by running the samples through ethanol 100\%: Spurr's resin-hard formulation $(75: 25 / \mathrm{V}: \mathrm{V})$ for $1 \mathrm{~h}$ at room temperature, then to ethanol 100\%: Spurr's resin-hard formulation $(50: 50 / \mathrm{V}: \mathrm{V})$ and finally to ethanol 100\%: Spurr's resin-hard formulation (25:75 / V:V) overnight. The following day the samples were transferred through 5 changes of 100\% Spurr's epoxy resin each $1 \mathrm{hr}$ long, and then placed in molds and polymerized for two days at $68^{\circ} \mathrm{C}$. Ultrathin sections (approx. $70 \mathrm{~nm}$ thick) were collected onto 200 mesh copper support grids and contrasted with Uranyl Acetate and Lead Citrate and then imaged using a CM 10 transmission electron microscope, under $100 \mathrm{Kv}$ accelerating voltage. Images were recorded with a Gatan Erlangshen CCD Digital camera. 


\section{ACKNOWLEDGEMENTS}

We are grateful to Mary Lou Guerinot and Jerry Kaplan for feedback on this manuscript. We also thank Jerry Kaplan for the yeast strains and plasmids used in this study, Liangtao Li for the yeast Western blot and help with aconitase assays, Elsbeth Walker for advice on the split root experiments, Inhwan Hwang for the pCamV3-GFP and $\mathrm{F}_{1}$-ATPase-RFP plasmids, James Chambers for assistance with confocal microscopy, Britney Privett for assistance with NanoString, Steve Eyles for help with ICP-MS, and Lara Strittmatter and UMass Medical School Electron Microscopy Facility for assistance with TEM. We also thank Veronica Voronina, Joye Yang, and Aleks Merkovich for technical assistance at various stages of this project.

This work was supported by the National Science Foundation (NSF) grant to JJ (IOS-1754969) and OKV (IOS-1656321 and IOS-1754966), the Gregory S. Call Undergraduate Research Program (LK, KT, EYP, FH, JG, MC, AK, DC, CD, EMP), Doelling Undergraduate Research Fund (LK, KT, EYP, JG, AK, DC, CD, EMP), Schupf Scholars Program (JZ), and the Sarles Fellowship (MC). The transmission electron microscopy was supported by the National Center for Research Resources award (SI0OD021580) to the UMass Medical School Electron Microscopy Facility. The authors are solely responsible for the content of this paper and do not necessarily represent the official views of the National Center for Research Resources, National Institutes of Health, or NSF. CHESS is supported by the NSF and NIH/NIGMS via NSF Award DMR-1332208. The authors declare no conflict of interests. 


\section{REFERENCES}

Alonso, J.M., Stepanova, A.N., Leisse, T.J., et al. (2003) Genome-wide insertional mutagenesis of Arabidopsis thaliana. Science, 301, 653-657.

Aranda, P.S., LaJoie, D.M. and Jorcyk, C.L. (2012) Bleach gel: a simple agarose gel for analyzing RNA quality. Electrophoresis, 33, 366-369.

Arnaud, N., Murgia, I., Boucherez, J., Briat, J.-F., Cellier, F. and Gaymard, F. (2006) An iron-induced nitric oxide burst precedes ubiquitin-dependent protein degradation for Arabidopsis AtFer1 ferritin gene expression. J. Biol. Chem., 281, 23579-23588.

Balk, J. and Schaedler, T.A. (2014) Iron cofactor assembly in plants. Annu. Rev. Plant Biol., 65, 125-153.

Barnes, J.D., Balaguer, L., Manrique, E., Elvira, S. and Davison, A.W. (1992) A reappraisal of the use of DMSO for the extraction and determination of chlorophylls a and $\mathrm{b}$ in lichens and higher plants. Environ Exp Bot, 32, 85-100.

Bashir, K., Ishimaru, Y., Shimo, H., et al. (2011) The rice mitochondrial iron transporter is essential for plant growth. Nat. Commun., 2, 322.

Bienfait, H.F., de Weger, L.A. and Kramer, D. (1987) Control of the development of ironefficiency reactions in potato as a response to iron deficiency is located in the roots. Plant Physiol., 83, 244-247.

Bogorad, L., Pires, G. and Swift, H. (1959) The structure of chloroplasts in leaf tissue of iron deficient Xanthium. Brookhaven Symp. Biol.

Bonaccorsi di Patti, M.C., Polticelli, F., Cece, G., Cutone, A., Felici, F., Persichini, T. and Musci, G. (2014) A structural model of human ferroportin and of its iron binding site. FEBS J., 281, 2851-2860.

Bonaccorsi di Patti, M.C., Polticelli, F., Tortosa, V., Furbetta, P.A. and Musci, G. (2015) A bacterial homologue of the human iron exporter ferroportin. FEBS Lett., 589, 3829-3835.

Briat, J.-F., Duc, C., Ravet, K. and Gaymard, F. (2010) Ferritins and iron storage in plants. Biochim. Biophys. Acta, 1800, 806-814.

Brink, S., Flügge, U.I., Chaumont, F., Boutry, M., Emmermann, M., Schmitz, U., Becker, K. and Pfanner, N. (1994) Preproteins of chloroplast envelope inner membrane contain targeting information for receptor-dependent import into fungal mitochondria. J. Biol. Chem., 269, 16478-16485.

Brumbarova, T., Bauer, P. and Ivanov, R. (2015) Molecular mechanisms governing Arabidopsis iron uptake. Trends Plant Sci., 20, 124-133. 
Buckhout, T.J., Yang, T.J.W. and Schmidt, W. (2009) Early iron-deficiency-induced transcriptional changes in Arabidopsis roots as revealed by microarray analyses. BMC Genomics, 10, 147.

Bughio, N., Takahashi, M., Yoshimura, E., Nishizawa, N.K. and Mori, S. (1997) LightDependent Iron Transport into Isolated Barley Chloroplasts. Plant and Cell Physiology, 38, 101-105.

Clemens, S. and Weber, M. (2016) The essential role of coumarin secretion for Fe acquisition from alkaline soil. Plant Signal. Behav., 11, e1114197.

Clough, S.J. and Bent, A.F. (1998) Floral dip: a simplified method for Agrobacterium-mediated transformation of Arabidopsis thaliana. Plant J., 16, 735-743.

Colombo, C., Palumbo, G., He, J.-Z., Pinton, R. and Cesco, S. (2014) Review on iron availability in soil: interaction of Fe minerals, plants, and microbes. J. Soils Sediments, 14, 538-548.

Connolly, E.L., Fett, J.P. and Guerinot, M.L. (2002) Expression of the IRT1 metal transporter is controlled by metals at the levels of transcript and protein accumulation. Plant Cell, 14, $1347-1357$.

Connorton, J.M., Balk, J. and Rodríguez-Celma, J. (2017) Iron homeostasis in plants - a brief overview. Metallomics, 9, 813-823.

Conte, S., Stevenson, D., Furner, I. and Lloyd, A. (2009) Multiple antibiotic resistance in Arabidopsis is conferred by mutations in a chloroplast-localized transport protein. Plant Physiol., 151, 559-573.

Conte, S.S., Chu, H.H., Rodriguez, D.C., Punshon, T., Vasques, K.A., Salt, D.E. and Walker, E.L. (2013) Arabidopsis thaliana Yellow Stripe1-Like4 and Yellow Stripe1Like6 localize to internal cellular membranes and are involved in metal ion homeostasis. Front. Plant Sci., 4, 283.

Conte, S.S. and Lloyd, A.M. (2010) The MAR1 transporter is an opportunistic entry point for antibiotics. Plant Signal. Behav., 5, 49-52.

Desbrosses-Fonrouge, A.-G., Voigt, K., Schröder, A., Arrivault, S., Thomine, S. and Krämer, U. (2005) Arabidopsis thaliana MTP1 is a Zn transporter in the vacuolar membrane which mediates $\mathrm{Zn}$ detoxification and drives leaf $\mathrm{Zn}$ accumulation. FEBS Lett., 579, 4165-4174.

DiDonato, R.J., Roberts, L.A., Sanderson, T., Eisley, R.B. and Walker, E.L. (2004) Arabidopsis Yellow Stripe-Like2 (YSL2): a metal-regulated gene encoding a plasma membrane transporter of nicotianamine-metal complexes. Plant J., 39, 403-414.

Dinneny, J.R., Long, T.A., Wang, J.Y., et al. (2008) Cell identity mediates the response of Arabidopsis roots to abiotic stress. Science, 320, 942-945. 
Divol, F., Couch, D., Conéjéro, G., Roschzttardtz, H., Mari, S. and Curie, C. (2013) The Arabidopsis YELLOW STRIPE LIKE4 and 6 transporters control iron release from the chloroplast. Plant Cell, 25, 1040-1055.

Drakesmith, H., Nemeth, E. and Ganz, T. (2015) Ironing out Ferroportin. Cell Metab., 22, $777-787$.

Durrett, T.P., Gassmann, W. and Rogers, E.E. (2007) The FRD3-mediated efflux of citrate into the root vasculature is necessary for efficient iron translocation. Plant Physiol., 144, 197-205.

Duy, D., Stübe, R., Wanner, G. and Philippar, K. (2011) The chloroplast permease PIC1 regulates plant growth and development by directing homeostasis and transport of iron. Plant Physiol., 155, 1709-1722.

Duy, D., Wanner, G., Meda, A.R., von Wirén, N., Soll, J. and Philippar, K. (2007) PIC1, an ancient permease in Arabidopsis chloroplasts, mediates iron transport. Plant Cell, 19, 986-1006.

Escudero, V., Abreu, I., Tejada-Jiménez, M., et al. (2020) Medicago truncatula Ferroportin2 mediates iron import into nodule symbiosomes. New Phytol.

Fourcroy, P., Sisó-Terraza, P., Sudre, D., et al. (2014) Involvement of the ABCG37 transporter in secretion of scopoletin and derivatives by Arabidopsis roots in response to iron deficiency. New Phytol., 201, 155-167.

Foury, F. and Roganti, T. (2002) Deletion of the mitochondrial carrier genes MRS3 and MRS4 suppresses mitochondrial iron accumulation in a yeast frataxin-deficient strain. J. Biol. Chem., 277, 24475-24483.

García, M.J., Romera, F.J., Stacey, M.G., Stacey, G., Villar, E., Alcántara, E. and PérezVicente, R. (2013) Shoot to root communication is necessary to control the expression of iron-acquisition genes in Strategy I plants. Planta, 237, 65-75.

Gomez-Casati, D.F., Busi, M.V. and Pagani, M.A. (2018) Plant frataxin in metal metabolism. Front. Plant Sci., 9, 1706.

Grusak, M.A. and Pezeshgi, S. (1996) Shoot-to-Root Signal Transmission Regulates Root Fe(III) Reductase Activity in the dgl Mutant of Pea. Plant Physiol., 110, 329-334.

Halliwell, B. and Gutteridge, J.M. (1992) Biologically relevant metal ion-dependent hydroxyl radical generation. An update. FEBS Lett., 307, 108-112.

Hantzis, L.J., Kroh, G.E., Jahn, C.E., Cantrell, M., Peers, G., Pilon, M. and Ravet, K. (2017) A program for iron economy during deficiency targets specific Fe proteins. Plant Physiol., 176, 596-610.

Heazlewood, J.L., Tonti-Filippini, J.S., Gout, A.M., Day, D.A., Whelan, J. and Millar, A.H. (2004) Experimental analysis of the Arabidopsis mitochondrial proteome highlights 
signaling and regulatory components, provides assessment of targeting prediction programs, and indicates plant-specific mitochondrial proteins. Plant Cell, 16, 241-256.

Hurt, E.C., Soltanifar, N., Goldschmidt-Clermont, M., Rochaix, J.D. and Schatz, G. (1986) The cleavable pre-sequence of an imported chloroplast protein directs attached polypeptides into yeast mitochondria. EMBO J., 5, 1343-1350.

Ichikawa, Y., Bayeva, M., Ghanefar, M., et al. (2012) Disruption of ATP-binding cassette B8 in mice leads to cardiomyopathy through a decrease in mitochondrial iron export. Proc. Natl. Acad. Sci. USA, 109, 4152-4157.

Ishka, M.R. and Vatamaniuk, O.K. (2020) Copper deficiency alters shoot architecture and reduces fertility of both gynoecium and androecium in Arabidopsis thaliana. BioRxiv.

Jain, A., Dashner, Z.S. and Connolly, E.L. (2019) Mitochondrial Iron Transporters (MIT1 and MIT2) Are Essential for Iron Homeostasis and Embryogenesis in Arabidopsis thaliana. Front. Plant Sci., 10, 1449.

Jaquinod, M., Villiers, F., Kieffer-Jaquinod, S., Hugouvieux, V., Bruley, C., Garin, J. and Bourguignon, J. (2007) A Proteomics Approach Highlights a Myriad of Transporters in the Arabidopsis thaliana Vacuolar Membrane. Plant Signal. Behav., 2, 413-415.

Jean, M. Le, Schikora, A., Mari, S., Briat, J.-F. and Curie, C. (2005) A loss-of-function mutation in AtYSL1 reveals its role in iron and nicotianamine seed loading. Plant J., 44, 769-782.

Jefferson, R.A., Kavanagh, T.A. and Bevan, M.W. (1987) GUS fusions: beta-glucuronidase as a sensitive and versatile gene fusion marker in higher plants. EMBO J., 6, 3901-3907.

Jeong, J., Cohu, C., Kerkeb, L., Pilon, M., Connolly, E.L. and Guerinot, M.L. (2008) Chloroplast $\mathrm{Fe}(\mathrm{III})$ chelate reductase activity is essential for seedling viability under iron limiting conditions. Proc. Natl. Acad. Sci. USA, 105, 10619-10624.

Jeong, J. and Connolly, E.L. (2009) Iron uptake mechanisms in plants: Functions of the FRO family of ferric reductases. Plant Sci., 176, 709-714.

Jeong, J., Merkovich, A., Clyne, M. and Connolly, E.L. (2017) Directing iron transport in dicots: regulation of iron acquisition and translocation. Curr. Opin. Plant Biol., 39, 106113.

Keech, O., Dizengremel, P. and Gardeström, P. (2005) Preparation of leaf mitochondria fromArabidopsis thaliana. Physiol. Plant., 124, 403-409.

Khan, M.A., Castro-Guerrero, N.A., McInturf, S.A., et al. (2018) Changes in iron availability in Arabidopsis are rapidly sensed in the leaf vasculature and impaired sensing leads to opposite transcriptional programs in leaves and roots. Plant Cell Environ., 41, 22632276. 
Kim, S.A., Punshon, T., Lanzirotti, A., Li, L., Alonso, J.M., Ecker, J.R., Kaplan, J. and Guerinot, M.L. (2006) Localization of iron in Arabidopsis seed requires the vacuolar membrane transporter VIT1. Science, 314, 1295-1298.

Klaumann, S., Nickolaus, S.D., Fürst, S.H., Starck, S., Schneider, S., Ekkehard Neuhaus, H. and Trentmann, O. (2011) The tonoplast copper transporter COPT5 acts as an exporter and is required for interorgan allocation of copper in Arabidopsis thaliana. New Phytol., 192, 393-404.

Kobayashi, T., Nozoye, T. and Nishizawa, N.K. (2018) Iron transport and its regulation in plants. Free Radic. Biol. Med., 133, 11-20.

Kumar, R.K., Chu, H.-H., Abundis, C., Vasques, K., Rodriguez, D.C., Chia, J.-C., Huang, R., Vatamaniuk, O.K. and Walker, E.L. (2017) Iron-Nicotianamine Transporters Are Required for Proper Long Distance Iron Signaling. Plant Physiol., 175, 1254-1268.

Lanquar, V., Lelièvre, F., Bolte, S., et al. (2005) Mobilization of vacuolar iron by AtNRAMP3 and AtNRAMP4 is essential for seed germination on low iron. EMBO J., 24, 4041-4051.

Lee, S.H., Singh, A.P., Chung, G.C., Kim, Y.S. and Kong, I.B. (2002) Chilling root temperature causes rapid ultrastructural changes in cortical cells of cucumber (Cucumis sativus L.) root tips. J. Exp. Bot., 53, 2225-2237.

Li, L., Miao, R., Bertram, S., Jia, X., Ward, D.M. and Kaplan, J. (2012) A role for ironsulfur clusters in the regulation of transcription factor Yap5-dependent high iron transcriptional responses in yeast. J. Biol. Chem., 287, 35709-35721.

Li, L., Miao, R., Jia, X., Ward, D.M. and Kaplan, J. (2014) Expression of the yeast cation diffusion facilitators $\mathrm{Mmt} 1$ and $\mathrm{Mmt} 2$ affects mitochondrial and cellular iron homeostasis: evidence for mitochondrial iron export. J. Biol. Chem., 289, 17132-17141.

Lin, H., Li, L., Jia, X., Ward, D.M. and Kaplan, J. (2011) Genetic and biochemical analysis of high iron toxicity in yeast: iron toxicity is due to the accumulation of cytosolic iron and occurs under both aerobic and anaerobic conditions. J. Biol. Chem., 286, 3851-3862.

López-Millán, A.-F., Grusak, M.A., Abadía, A. and Abadía, J. (2013) Iron deficiency in plants: an insight from proteomic approaches. Front. Plant Sci., 4, 254.

Mai, H.-J., Pateyron, S. and Bauer, P. (2016) Iron homeostasis in Arabidopsis thaliana: transcriptomic analyses reveal novel FIT-regulated genes, iron deficiency marker genes and functional gene networks. BMC Plant Biol., 16, 211.

Marschner, P. ed. (2012) Marschner's Mineral Nutrition of Higher Plants 3rd ed.,.

Masuda, T., Suzuki, T., Shimada, H., Ohta, H. and Takamiya, K. (2003) Subcellular localization of two types of ferrochelatase in cucumber. Planta.

Mendoza-Cózatl, D.G., Xie, Q., Akmakjian, G.Z., et al. (2014) OPT3 is a component of the iron-signaling network between leaves and roots and misregulation of OPT3 leads to an over-accumulation of cadmium in seeds. Mol. Plant, 7, 1455-1469. 
Merlot, S., Hannibal, L., Martins, S., Martinelli, L., Amir, H., Lebrun, M. and Thomine, S. (2014) The metal transporter PgIREG1 from the hyperaccumulator Psychotria gabriellae is a candidate gene for nickel tolerance and accumulation. J. Exp. Bot., 65, 1551-1564.

Metzendorf, C., Wu, W. and Lind, M.I. (2009) Overexpression of Drosophila mitoferrin in 1(2)mbn cells results in dysregulation of Fer1HCH expression. Biochem. J., 421, 463471.

Morrissey, J., Baxter, I.R., Lee, J., Li, L., Lahner, B., Grotz, N., Kaplan, J., Salt, D.E. and Guerinot, M.L. (2009) The ferroportin metal efflux proteins function in iron and cobalt homeostasis in Arabidopsis. Plant Cell, 21, 3326-3338.

Mühlenhoff, U., Stadler, J.A., Richhardt, N., Seubert, A., Eickhorst, T., Schweyen, R.J., Lill, R. and Wiesenberger, G. (2003) A specific role of the yeast mitochondrial carriers MRS3/4p in mitochondrial iron acquisition under iron-limiting conditions. J. Biol. Chem., 278, 40612-40620.

Mukherjee, I., Campbell, N.H., Ash, J.S. and Connolly, E.L. (2006) Expression profiling of the Arabidopsis ferric chelate reductase (FRO) gene family reveals differential regulation by iron and copper. Planta, 223, 1178-1190.

Müller, B., Kovács, K., Pham, H.-D., et al. (2019) Chloroplasts preferentially take up ferriccitrate over iron-nicotianamine complexes in Brassica napus. Planta, 249, 751-763.

Nakagawa, T., Suzuki, T., Murata, S., et al. (2007) Improved Gateway binary vectors: highperformance vectors for creation of fusion constructs in transgenic analysis of plants. Biosci. Biotechnol. Biochem., 71, 2095-2100.

Nelson, B.K., Cai, X. and Nebenführ, A. (2007) A multicolored set of in vivo organelle markers for co-localization studies in Arabidopsis and other plants. Plant J., 51, 11261136.

Notredame, C., Higgins, D.G. and Heringa, J. (2000) T-Coffee: A novel method for fast and accurate multiple sequence alignment. J. Mol. Biol., 302, 205-217.

Paradkar, P.N., Zumbrennen, K.B., Paw, B.H., Ward, D.M. and Kaplan, J. (2009)

Regulation of mitochondrial iron import through differential turnover of mitoferrin 1 and mitoferrin 2. Mol. Cell. Biol., 29, 1007-1016.

Park, E.Y., Tsuyuki, K.M., Hu, F., Lee, J. and Jeong, J. (2019) PRC2-Mediated H3K27me3 Contributes to Transcriptional Regulation of FIT-Dependent Iron Deficiency Response. Front. Plant Sci., 10, 627.

Pascal, N. and Douce, R. (1993) Effect of Iron Deficiency on the Respiration of Sycamore (Acer pseudoplatanus L.) Cells. Plant Physiol., 103, 1329-1338.

Petit, J.M., Briat, J.F. and Lobréaux, S. (2001) Structure and differential expression of the four members of the Arabidopsis thaliana ferritin gene family. Biochem. J., 359, 575582. 
Pfaller, R., Pfanner, N. and Neupert, W. (1989) Mitochondrial protein import. Bypass of proteinaceous surface receptors can occur with low specificity and efficiency. J. Biol. Chem., 264, 34-39.

Pham, H.D., Pólya, S., Müller, B., et al. (2020) The developmental and iron nutritional pattern of PIC1 and NiCo does not support their interdependent and exclusive collaboration in chloroplast iron transport in Brassica napus. Planta, 251, 96.

Platt-Aloia, K.A., Thomson, W.W. and Terry, N. (1983) Changes in plastid ultrastructure during iron nutrition-mediated chloroplast development. Protoplasma, 114-114, 85-92.

Porra, R.J., Thompson, W.A. and Kriedemann, P.E. (1989) Determination of accurate extinction coefficients and simultaneous equations for assaying chlorophylls $\mathrm{a}$ and $\mathrm{b}$ extracted with four different solvents: verification of the concentration of chlorophyll standards by atomic absorption spectroscopy. Biochimica et Biophysica Acta (BBA) Bioenergetics, 975, 384-394.

Raven, J.A. and Allen, J.F. (2003) Genomics and chloroplast evolution: what did cyanobacteria do for plants? Genome Biol., 4, 209.

Ravet, K., Touraine, B., Boucherez, J., Briat, J.-F., Gaymard, F. and Cellier, F. (2009) Ferritins control interaction between iron homeostasis and oxidative stress in Arabidopsis. Plant J., 57, 400-412.

Regev-Rudzki, N., Karniely, S., Ben-Haim, N.N. and Pines, O. (2005) Yeast aconitase in two locations and two metabolic pathways: seeing small amounts is believing. Mol. Biol. Cell, 16, 4163-4171.

Ren, Y., Yang, S., Tan, G., et al. (2012) Reduction of mitoferrin results in abnormal development and extended lifespan in Caenorhabditis elegans. PLoS One, 7, e29666.

Robinson, N.J., Procter, C.M., Connolly, E.L. and Guerinot, M.L. (1999) A ferric-chelate reductase for iron uptake from soils. Nature, 397, 694-697.

Rockwell, N.C., Lagarias, J.C. and Bhattacharya, D. (2014) Primary endosymbiosis and the evolution of light and oxygen sensing in photosynthetic eukaryotes. Front. Ecol. Evol., 2.

Rodríguez-Celma, J., Pan, I.C., Li, W., Lan, P., Buckhout, T.J. and Schmidt, W. (2013) The transcriptional response of Arabidopsis leaves to Fe deficiency. Front. Plant Sci., 4, 276.

Roger, A.J., Muñoz-Gómez, S.A. and Kamikawa, R. (2017) The origin and diversification of mitochondria. Curr. Biol., 27, R1177-R1192.

Santi, S. and Schmidt, W. (2009) Dissecting iron deficiency-induced proton extrusion in Arabidopsis roots. New Phytol., 183, 1072-1084.

Schaaf, G., Honsbein, A., Meda, A.R., Kirchner, S., Wipf, D. and von Wirén, N. (2006) AtIREG2 encodes a tonoplast transport protein involved in iron-dependent nickel detoxification in Arabidopsis thaliana roots. J. Biol. Chem., 281, 25532-25540. 
Schaaf, G., Schikora, A., Häberle, J., Vert, G., Ludewig, U., Briat, J.-F., Curie, C. and von Wirén, N. (2005) A putative function for the arabidopsis Fe-Phytosiderophore transporter homolog AtYSL2 in Fe and Zn homeostasis. Plant Cell Physiol., 46, 762774.

Schmid, M., Davison, T.S., Henz, S.R., Pape, U.J., Demar, M., Vingron, M., Schölkopf, B., Weigel, D. and Lohmann, J.U. (2005) A gene expression map of Arabidopsis thaliana development. Nat. Genet., 37, 501-506.

Schmidt, H., Günther, C., Weber, M., Spörlein, C., Loscher, S., Böttcher, C., Schobert, R. and Clemens, S. (2014) Metabolome analysis of Arabidopsis thaliana roots identifies a key metabolic pathway for iron acquisition. PLoS One, 9, e102444.

Schmittgen, T.D. and Livak, K.J. (2008) Analyzing real-time PCR data by the comparative CT method. Nat. Protoc., 3, 1101-1108.

Schwacke, R., Schneider, A., van der Graaff, E., Fischer, K., Catoni, E., Desimone, M., Frommer, W.B., Flügge, U.-I. and Kunze, R. (2003) ARAMEMNON, a novel database for Arabidopsis integral membrane proteins. Plant Physiol., 131, 16-26.

Shaw, G.C., Cope, J.J., Li, L., et al. (2006) Mitoferrin is essential for erythroid iron assimilation. Nature, 440, 96-100.

Shcolnick, S. and Keren, N. (2006) Metal homeostasis in cyanobacteria and chloroplasts. Balancing benefits and risks to the photosynthetic apparatus. Plant Physiol., 141, 805810.

Shikanai, T., Müller-Moulé, P., Munekage, Y., Niyogi, K.K. and Pilon, M. (2003) PAA1, a P-type ATPase of Arabidopsis, functions in copper transport in chloroplasts. Plant Cell, 15, 1333-1346.

Shimoni-Shor, E., Hassidim, M., Yuval-Naeh, N. and Keren, N. (2010) Disruption of Nap14, a plastid-localized non-intrinsic ABC protein in Arabidopsis thaliana results in the overaccumulation of transition metals and in aberrant chloroplast structures. Plant Cell Environ., 33, 1029-1038.

Shingles, R., North, M. and McCarty, R.E. (2002) Ferrous ion transport across chloroplast inner envelope membranes. Plant Physiol., 128, 1022-1030.

Smith, M.D., Schnell, D.J., Fitzpatrick, L. and Keegstra, K. (2003) In vitro analysis of chloroplast protein import. Curr. Protoc. Cell Biol., Chapter 11, Unit11.16.

Solé, V.A., Papillon, E., Cotte, M., Walter, P. and Susini, J. (2007) A multiplatform code for the analysis of energy-dispersive X-ray fluorescence spectra. Spectrochimica Acta Part B At. Spectrosc., 62, 63-68.

Solti, A., Kovács, K., Basa, B., Vértes, A., Sárvári, E. and Fodor, F. (2012) Uptake and incorporation of iron in sugar beet chloroplasts. Plant Physiol. Biochem., 52, 91-97. 
Stacey, M.G., Patel, A., McClain, W.E., Mathieu, M., Remley, M., Rogers, E.E., Gassmann, W., Blevins, D.G. and Stacey, G. (2008) The Arabidopsis AtOPT3 protein functions in metal homeostasis and movement of iron to developing seeds. Plant Physiol., 146, 589601.

Stocking, C.R. (1975) Iron Deficiency and the Structure and Physiology of Maize Chloroplasts. Plant Physiol., 55, 626-631.

Szabo, I. and Zoratti, M. (2014) Mitochondrial channels: ion fluxes and more. Physiol. Rev., 94, 519-608.

Tan, Y.-F., O'Toole, N., Taylor, N.L. and Millar, A.H. (2010) Divalent metal ions in plant mitochondria and their role in interactions with proteins and oxidative stress-induced damage to respiratory function. Plant Physiol., 152, 747-761.

Tanaka, R., Kobayashi, K. and Masuda, T. (2011) Tetrapyrrole Metabolism in Arabidopsis thaliana. Arabidopsis Book, 9, e0145.

Taniguchi, R., Kato, H.E., Font, J., Deshpande, C.N., Wada, M., Ito, K., Ishitani, R., Jormakka, M. and Nureki, O. (2015) Outward- and inward-facing structures of a putative bacterial transition-metal transporter with homology to ferroportin. Nat. Commun., 6, 8545.

Tarantino, D., Casagrande, F., Soave, C. and Murgia, I. (2010) Knocking out of the mitochondrial AtFer4 ferritin does not alter response of Arabidopsis plants to abiotic stresses. J. Plant Physiol., 167, 453-460.

Tarantino, D., Morandini, P., Ramirez, L., Soave, C. and Murgia, I. (2011) Identification of an Arabidopsis mitoferrinlike carrier protein involved in Fe metabolism. Plant Physiol. Biochem., 49, 520-529.

Tarantino, D., Santo, N., Morandini, P., Casagrande, F., Braun, H.-P., Heinemeyer, J., Vigani, G., Soave, C. and Murgia, I. (2010) AtFer4 ferritin is a determinant of iron homeostasis in Arabidopsis thaliana heterotrophic cells. J. Plant Physiol., 167, 15981605.

Teardo, E., Carraretto, L., Bortoli, S. De, Costa, A., Behera, S., Wagner, R., Schiavo, F. Lo, Formentin, E. and Szabo, I. (2015) Alternative splicing-mediated targeting of the Arabidopsis GLUTAMATE RECEPTOR3.5 to mitochondria affects organelle morphology. Plant Physiol., 167, 216-227.

Terry, N. and Low, G. (1982) Leaf chlorophyll content and its relation to the intracellular localization of iron. J. Plant Nutr., 5, 301-310.

Thimm, O., Essigmann, B., Kloska, S., Altmann, T. and Buckhout, T.J. (2001) Response of Arabidopsis to iron deficiency stress as revealed by microarray analysis. Plant Physiol., 127, 1030-1043. 
Thomine, S., Lelièvre, F., Debarbieux, E., Schroeder, J.I. and Barbier-Brygoo, H. (2003) AtNRAMP3, a multispecific vacuolar metal transporter involved in plant responses to iron deficiency. Plant J., 34, 685-695.

Varotto, C., Maiwald, D., Pesaresi, P., Jahns, P., Salamini, F. and Leister, D. (2002) The metal ion transporter IRT1 is necessary for iron homeostasis and efficient photosynthesis in Arabidopsis thaliana. Plant J., 31, 589-599.

Vartapetian, B.B., Andreeva, I. and Generozova, I.P. (2003) Functional electron microscopy in studies of plant response and adaptation to anaerobic stress. Annals of ....

Vasconcelos, M.W., Gruissem, W. and Bhullar, N.K. (2017) Iron biofortification in the 21st century: setting realistic targets, overcoming obstacles, and new strategies for healthy nutrition. Curr. Opin. Biotechnol., 44, 8-15.

Versaw, W.K. and Harrison, M.J. (2002) A chloroplast phosphate transporter, PHT2;1, influences allocation of phosphate within the plant and phosphate-starvation responses. Plant Cell, 14, 1751-1766.

Vert, G., Grotz, N., Dédaldéchamp, F., Gaymard, F., Guerinot, M.L., Briat, J.-F. and Curie, C. (2002) IRT1, an Arabidopsis transporter essential for iron uptake from the soil and for plant growth. Plant Cell, 14, 1223-1233.

Vert, G.A., Briat, J.-F. and Curie, C. (2003) Dual regulation of the Arabidopsis high-affinity root iron uptake system by local and long-distance signals. Plant Physiol., 132, 796-804.

Vigani, G., Faoro, F., Ferretti, A.M., Cantele, F., Maffi, D., Marelli, M., Maver, M., Murgia, I. and Zocchi, G. (2015) Three-Dimensional Reconstruction, by TEM Tomography, of the Ultrastructural Modifications Occurring in Cucumis sativus L. Mitochondria under Fe Deficiency. PLoS One, 10, e0129141.

Voith von Voithenberg, L., Park, J., Stübe, R., Lux, C., Lee, Y. and Philippar, K. (2019) A Novel Prokaryote-Type ECF/ABC Transporter Module in Chloroplast Metal Homeostasis. Front. Plant Sci., 10, 1264.

Waters, B.M., Chu, H.-H., Didonato, R.J., Roberts, L.A., Eisley, R.B., Lahner, B., Salt, D.E. and Walker, E.L. (2006) Mutations in Arabidopsis yellow stripe-likel and yellow stripelike3 reveal their roles in metal ion homeostasis and loading of metal ions in seeds. Plant Physiol., 141, 1446-1458.

Winter, D., Vinegar, B., Nahal, H., Ammar, R., Wilson, G.V. and Provart, N.J. (2007) An "Electronic Fluorescent Pictograph" browser for exploring and analyzing large-scale biological data sets. PLoS One, 2, e718.

Yang, T.J.W., Lin, W.-D. and Schmidt, W. (2010) Transcriptional profiling of the Arabidopsis iron deficiency response reveals conserved transition metal homeostasis networks. Plant Physiol., 152, 2130-2141. 
Yokosho, K., Yamaji, N., Mitani-Ueno, N., Shen, R.F. and Ma, J.F. (2016) An AluminumInducible IREG Gene is Required for Internal Detoxification of Aluminum in Buckwheat. Plant Cell Physiol., 57, 1169-1178.

Yoon, H.S., Hackett, J.D., Ciniglia, C., Pinto, G. and Bhattacharya, D. (2004) A molecular timeline for the origin of photosynthetic eukaryotes. Mol. Biol. Evol., 21, 809-818.

Yoshinaga, K., Arimura, S.I., Niwa, Y. and Tsutsumi, N. (2005) Mitochondrial Behaviour in the Early Stages of ROS Stress Leading to Cell Death in Arabidopsis thaliana. Annals of ....

Zancani, M., Peresson, C., Biroccio, A., et al. (2004) Evidence for the presence of ferritin in plant mitochondria. Eur. J. Biochem., 271, 3657-3664.

Zhai, Z., Gayomba, S.R., Jung, H.-I., et al. (2014) OPT3 Is a Phloem-Specific Iron Transporter That Is Essential for Systemic Iron Signaling and Redistribution of Iron and Cadmium in Arabidopsis. Plant Cell, 26, 2249-2264.

Zhang, X.-Y., Zhang, X., Zhang, Q., Pan, X.-X., Yan, L.-C., Ma, X.-J., Zhao, W.-Z., Qi, X.T. and Yin, L.-P. (2017) Zea mays Fe deficiency-related 4 (ZmFDR4) functions as an iron transporter in the plastids of monocots. Plant J., 90, 147-163.

Zhang, Y., Xu, Y.-H., Yi, H.-Y. and Gong, J.-M. (2012) Vacuolar membrane transporters OsVIT1 and OsVIT2 modulate iron translocation between flag leaves and seeds in rice. Plant J., 72, 400-410.

Zimmermann, P., Hirsch-Hoffmann, M., Hennig, L. and Gruissem, W. (2004) GENEVESTIGATOR. Arabidopsis microarray database and analysis toolbox. Plant Physiol., 136, 2621-2632.

Zimorski, V., Ku, C., Martin, W.F. and Gould, S.B. (2014) Endosymbiotic theory for organelle origins. Curr. Opin. Microbiol., 22, 38-48. 


\section{FIGURE LEGENDS}

Figure 1. Sequence alignment of FPNs. Multiple sequence alignment was performed using TCoffee (Notredame et al., 2000) with FPN amino acid sequences of Arabidopsis thaliana (AtFPN1, AtFPN2, AtFPN3), Homo sapiens (HsFPN), Mus musculus (MmFPN), Danio reio (DrFPN), and Bdellovibrio bacteriovorus (BbFPN). Identical residues are shaded in black, whereas similar residues are shaded in grey. Red stars indicate residues involved in iron binding and transport in human and bacterial FPNs (Bonaccorsi di Patti et al., 2015; Taniguchi et al., 2015). The blue stars indicate motif $A, G_{3} D X_{3} R$, which is conserved in MFS members. The predicted transit peptide, denoted as a blue bar, suggests a chloroplast or mitochondrial localization (Schwacke et al., 2003). The yellow brown bars indicate predicted transmembrane domains.

Figure 2. Heterologous expression of FPN3 in yeast. (A) Spot assays in high iron media (SDLeu with $2 \mathrm{mM}$ and $3 \mathrm{mM} \mathrm{Fe}$ ) or control media (SD-Leu) with $\Delta c c c t 1 \Delta m m t 1 / 2$ cells expressing FPN3 or MMT1/2, or transformed with an empty vector, $p R S 315$. (B) Growth assays with liquid culture with $\triangle c c c t 1 \Delta m m t 1 / 2$ cells expressing $F P N 3$ or $M M T 1 / 2$, or transformed with an empty vector, $p R S 315$. Significant differences compared to the negative control were determined by two-way ANOVA followed by Tukey's multiple comparison test $\left(^{*}: \mathrm{p}<0.05 ;{ }^{*}\right.$ : $\mathrm{p}<0.001 ; \mathrm{n}=6$; Error bars $=\mathrm{SD}$ ). From the third time point and beyond, cells expressing FPN3 or MMT1/2 were significantly different from the negative control $(\mathrm{p}<0.001)$ but labels were omitted for simplicity of the graph. (C) Aconitase activity (nmol/mg protein/min) of wild type (DY150) cells expressing $F P N 3$ or $M M T 1 / 2$ or transformed with an empty vector, $p R S 426(\mathrm{n}=6$; Error bars $=$ SD). (D) cGDO activity ( $\mathrm{mmol} / \mathrm{mg}$ protein/min) of wild type (DY150) cells expressing FPN3, 
$M M T 1 / 2$, or the empty vector, $p R S 315$. Significant differences were determined by one-way ANOVA followed by Tukey's test $(*: \mathrm{p}<0.01 ; \mathrm{n}=6$; Error bars $=\mathrm{SD})$.

Figure 3. FPN3 expression detected by FPN3p-GUS and RT-qPCR. (A-C) Histochemical staining of FPN3p-GUS seedlings germinated under iron sufficient conditions on day 3 (A) and day 5 (C), and a close-up image of the root tip of a day 3 seedling (B; boxed region in A). (D) FPN3p-GUS expression in the first true leaf of a 3-week old plant. (E, F) FPN3p-GUS staining in floral organs (E) and the silique (F). (G, H) FPN3p-GUS staining in 1-day-old imbibing seedlings germinated on iron deficient $(\mathrm{G})$ or sufficient $(\mathrm{H})$ media. (I) FPN3p-GUS staining in 3day-old seedlings germinated on iron deficient (-Fe) or sufficient (+Fe) media. (J, K) Close-up view of iron sufficient root $(\mathrm{J})$ and iron deficient root $(\mathrm{K})$. Representative images of seedlings or organs from at least 6 individuals are shown. (L) Relative expression of FPN3 in shoots and roots of wild type plants treated under iron deficient or sufficient conditions. FPN3 transcript level was quantified by RT-qPCR and normalized with ACT2. (ANOVA; Tukey's test; *: $\mathrm{p}<0.05 ; \mathrm{n}=3$; error bars $=\mathrm{SD})$.

Figure 4. Steady state FPN3 transcript levels in split-roots. (A) Schematic overview of iron growth conditions prior to RNA extraction. (B) FPN3 transcript levels in split roots. Seedlings were grown under iron-deficient $(-\mathrm{Fe})$ or iron-sufficient $(+\mathrm{Fe})$ conditions, then each root was treated appropriately with iron-deficient or iron-sufficient media for 0,6 , or 12 hours. The iron growth conditions of each split root are denoted as: $+\mathrm{Fe} /+\mathrm{Fe}$ for growth in iron sufficient conditions followed by iron sufficiency treatment; $+\mathrm{Fe} /-\mathrm{Fe}$ for growth in iron sufficient conditions followed by iron deficiency treatment; -Fe/+Fe for growth in iron deficient conditions followed by iron sufficiency treatment; -Fe/-Fe for growth in iron deficient conditions followed 
by iron deficiency treatment. Significant differences compared to 0 hour were determined by one-way ANOVA followed by Dunnett's test $\left(*\right.$ : $\mathrm{p}<0.05 ; *^{*}: \mathrm{p}<0.01 ; \mathrm{n}=5$; error bars $\left.=\mathrm{SD}\right)$.

Figure 5. Growth of $f p n 3$ under iron deficient conditions. (A) Wild type (Col 0) and fpn3 single mutants germinated and grown in control soil or alkaline soil $(\sim \mathrm{pH} 8)$ with or without iron irrigation. (B) Quantified shoot fresh weights of wild type (Col 0), fpn3 single mutants, germinated and grown in control soil or alkaline soil. Mean values of 36 to 40 individuals are shown. Statistically significant differences compared to Col 0 are denoted (One-way ANOVA; Dunnett's test; *: $\mathrm{p}<0.05$; error bars $=\mathrm{SD}$ ).

Figure 6. Elemental analysis of $f p n 3$ shoots and roots (A) Iron content of shoot tissue from plants grown under iron sufficient $(+\mathrm{Fe})$ and deficient $(-\mathrm{Fe})$ conditions measured by ICP-MS. (B) Iron content of root tissue from plants grown under iron sufficient $(+\mathrm{Fe})$ and deficient $(-\mathrm{Fe})$ conditions measured by ICP-MS. Mean values of pooled quadruplicate ICP-MS samples are shown with error bars (SE). Significant differences compared to Col 0 are denoted (One-way ANOVA; Dunnett's test; *: $\mathrm{p}<0.05 ; * *: \mathrm{p}<0.01$ ). (C) Synchrotron $\mathrm{x}$-ray fluorescence microscopy images of iron distribution in leaves of wild type $(\mathrm{Col} \mathrm{0})$ and fpn3 leaves. The first true leaves of 22-day old plants grown in iron sufficient conditions were imaged at a resolution of $30 \times 30 \mu \mathrm{m}$ with $0.2 \mathrm{sec}$ dwell time. Representative images of leaves from three individuals are shown. Scale bars $=1 \mathrm{~mm}$.

\section{Figure 7. Subcellular localization of FPN3-GFP and elemental analysis of $f p n 3$}

mitochondria and chloroplasts. (A-C) Confocal image of an optical section of Arabidopsis root stably expressing FPN3-GFP (A) and stained with MitoTracker Red (B). (C) Overlay image of 
(A) and (B) shows the co-localization of FPN3-GFP with MitoTracker Red. (D-F) Confocal image of an optical section of Arabidopsis root stably co-expressing FPN3-GFP (D) and pt-RFP (E). (F) Overlay image of (D) and (E) shows the co-localization of FPN3-GFP with $p t-R F P$. Scale bars $=25 \mu \mathrm{m} .(\mathrm{G}, \mathrm{H})$ Elemental analysis of chloroplasts and mitochondria isolated from fpn3 shoots. (G) Iron content of chloroplasts measured by ICP-MS and normalized with chlorophyll content. $(\mathrm{H})$ Iron content of mitochondria normalized with mitochondrial protein. Mean values of quadruplicate chloroplast samples and triplicate mitochondrial samples are shown with error bars (SD). Significant differences compared to the wild type, Col 0, are denoted (One-way ANOVA followed by Dunnett's test; *: $\mathrm{p}<0.05$ ).

Figure 8. Expression of organellar iron homeostasis genes in $f p n 3$ shoots. Normalized steady-state level transcripts detected by NanoString. Seedlings were grown in B5 without sucrose for 2 weeks and transferred to iron-deficient or iron-sufficient media for 3 days. Mean values of normalized transcript levels are shown. Significant differences compared to Col 0 samples from the same treatment are denoted (One-way ANOVA; Dunnett's test; *: $\mathrm{p}<0.05$; $\mathrm{n}=4$; Error bars $=\mathrm{SD}$ ).

\section{Figure 9. Mitochondria and chloroplast ultrastructure of $f p n 3$.}

(A) TEM images of the wild type, Col 0, fpn3-1, and fpn3-2 leaf sections with mesophyll cells from iron sufficient conditions. Close-up images of mitochondria (middle row) and thylakoids (bottom row) are shown. (B) Area of mitochondria from iron sufficient conditions. data point represents the quantified area from each mitochondrion section, and the lines represent mean values with SD. Ninety-four to 105 mitochondria from 38 to 41 cells were quantified. (C) TEM images of iron deficient wild type, fpn3-1, and fpn3-2 leaf sections with mesophyll cells. 
Chloroplasts (c) and mitochondria (m) are labeled in the images. Scale bars $=200 \mathrm{~nm}$. (D) Area of mitochondria from iron deficient conditions. Each data point represents the quantified area from each mitochondrion section, and the lines represent mean values with SD. Ninety-six to 113 mitochondria from 40 to 57 cells were quantified. (one-way ANOVA; Tukey's multiple comparison test' $*$ : $\mathrm{p}^{<0.01)}$. 
bioRxiv preprint doi: https://doi.org/10.1101/2020.07.15.203646; this version posted November 7, 2020. The copyright holder for this preprint (which was not certified by peer review) is the author/funder, who has granted bioRxiv a license to display the preprint in perpetuity. It is made available under aCC-BY 4.0 International license.

Figure 1.
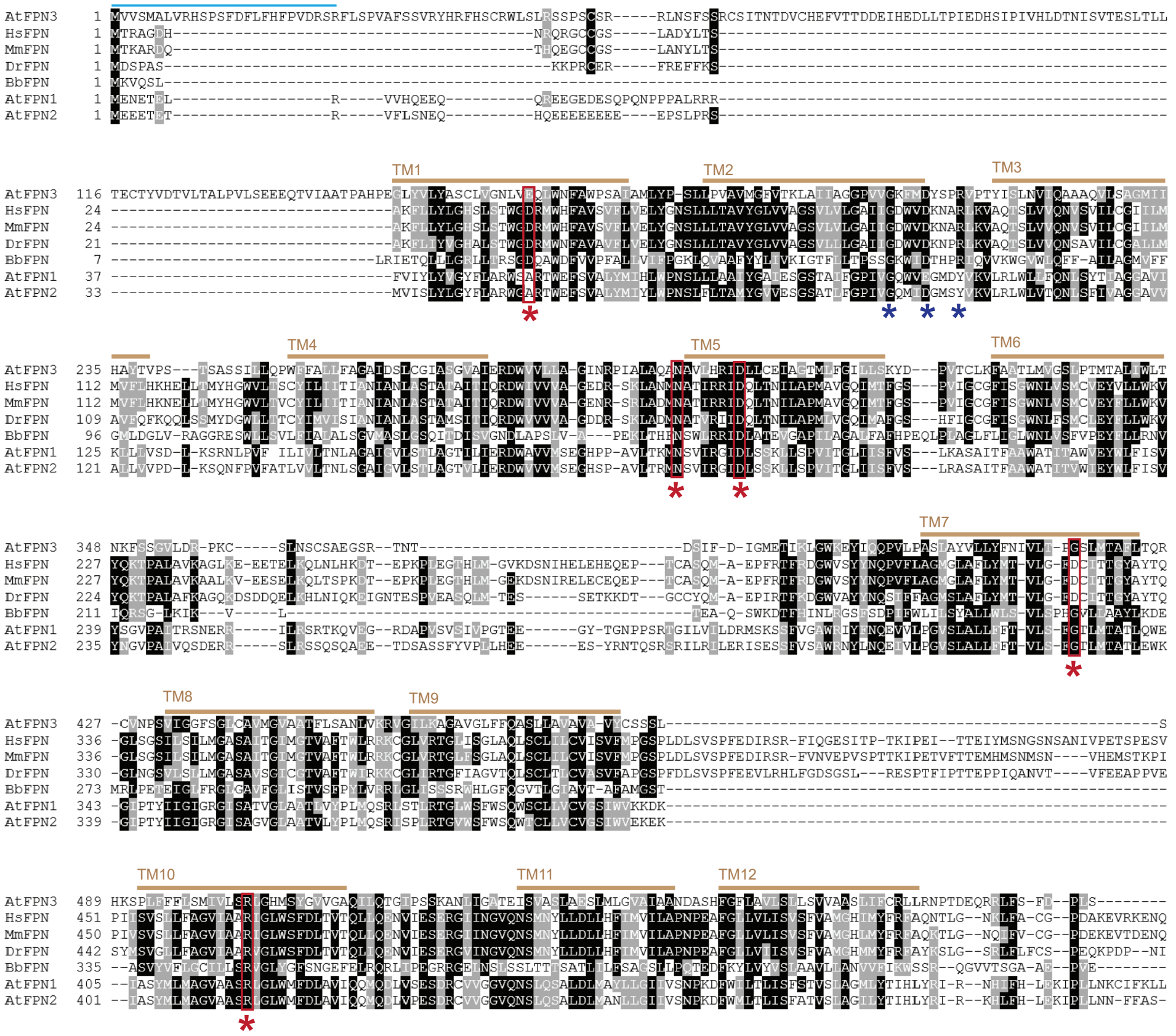

\footnotetext{
AtFPN3 $598------N$

HSFPN 566 AN-TSVV

MmFPN 565 PN-TSVV

DIFPN 556 PSLPNSV

BbFPN $440------S$

AtFPN1 519 PS-RGNV
} 


\section{Figure 2.}

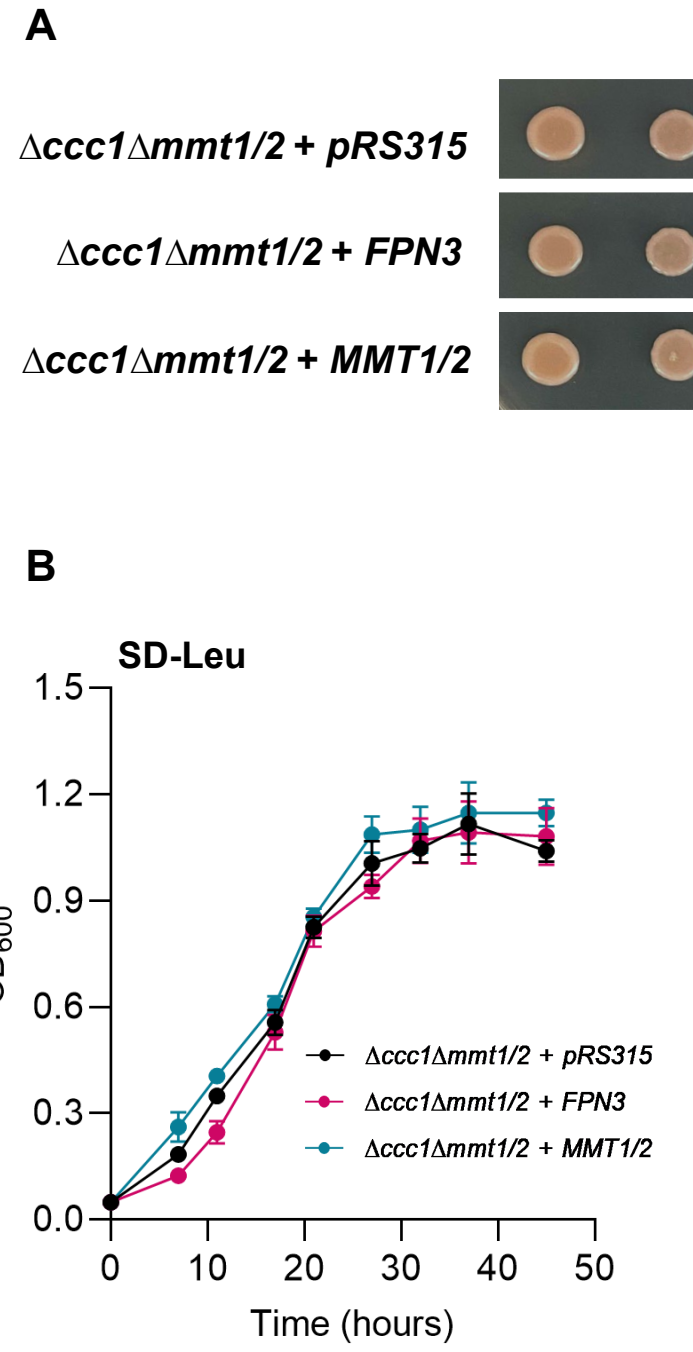

SD-Leu

(1) 80

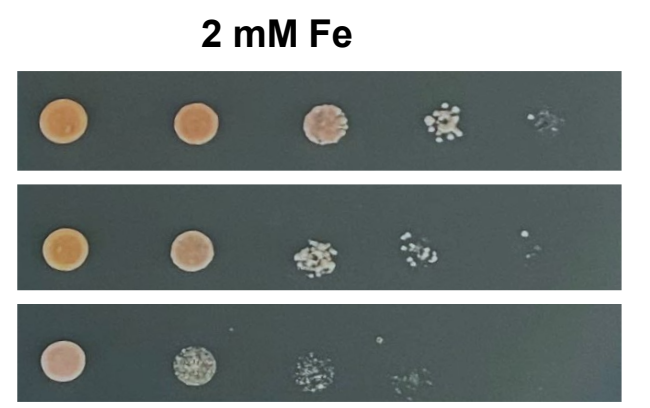

C

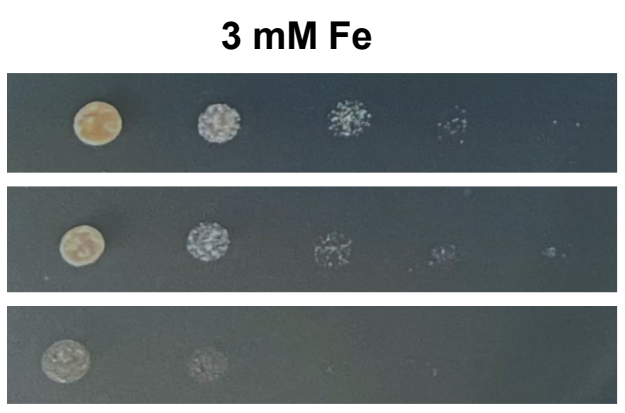

D
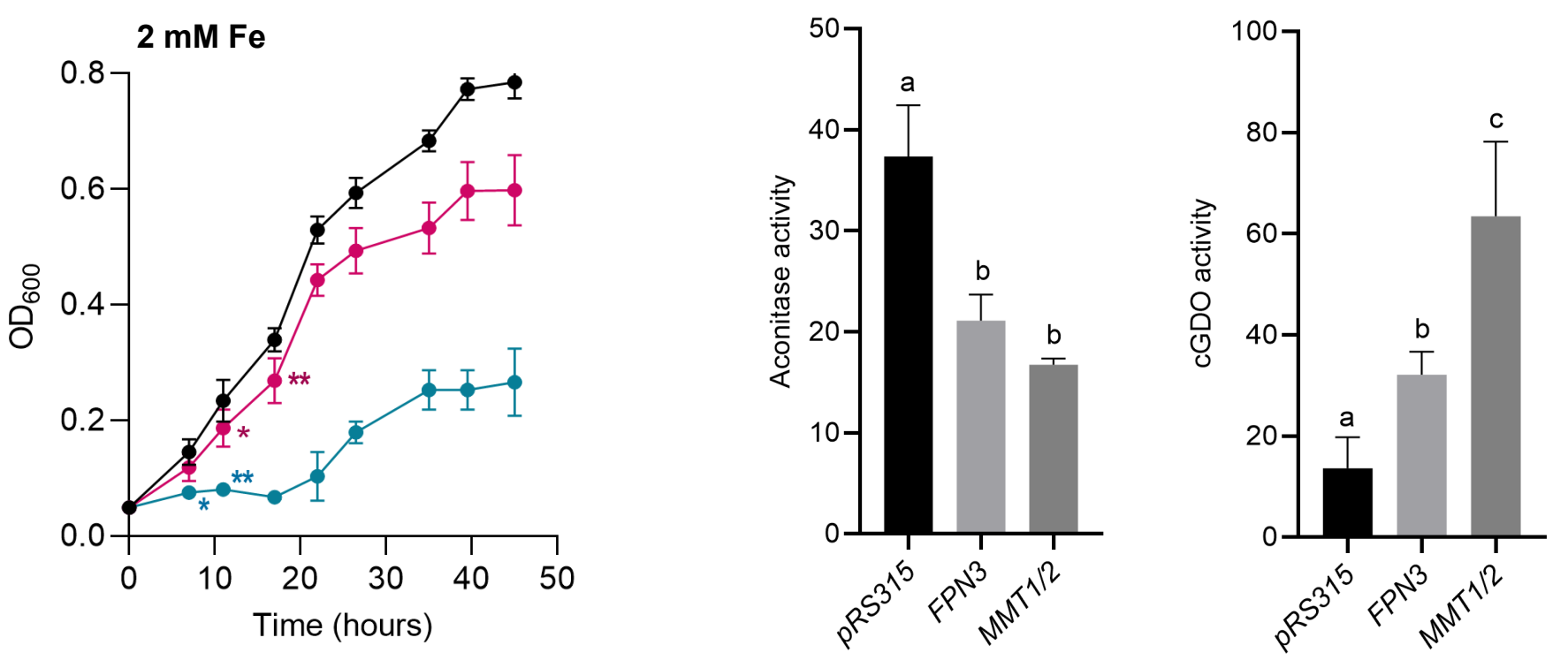
Figure 3.
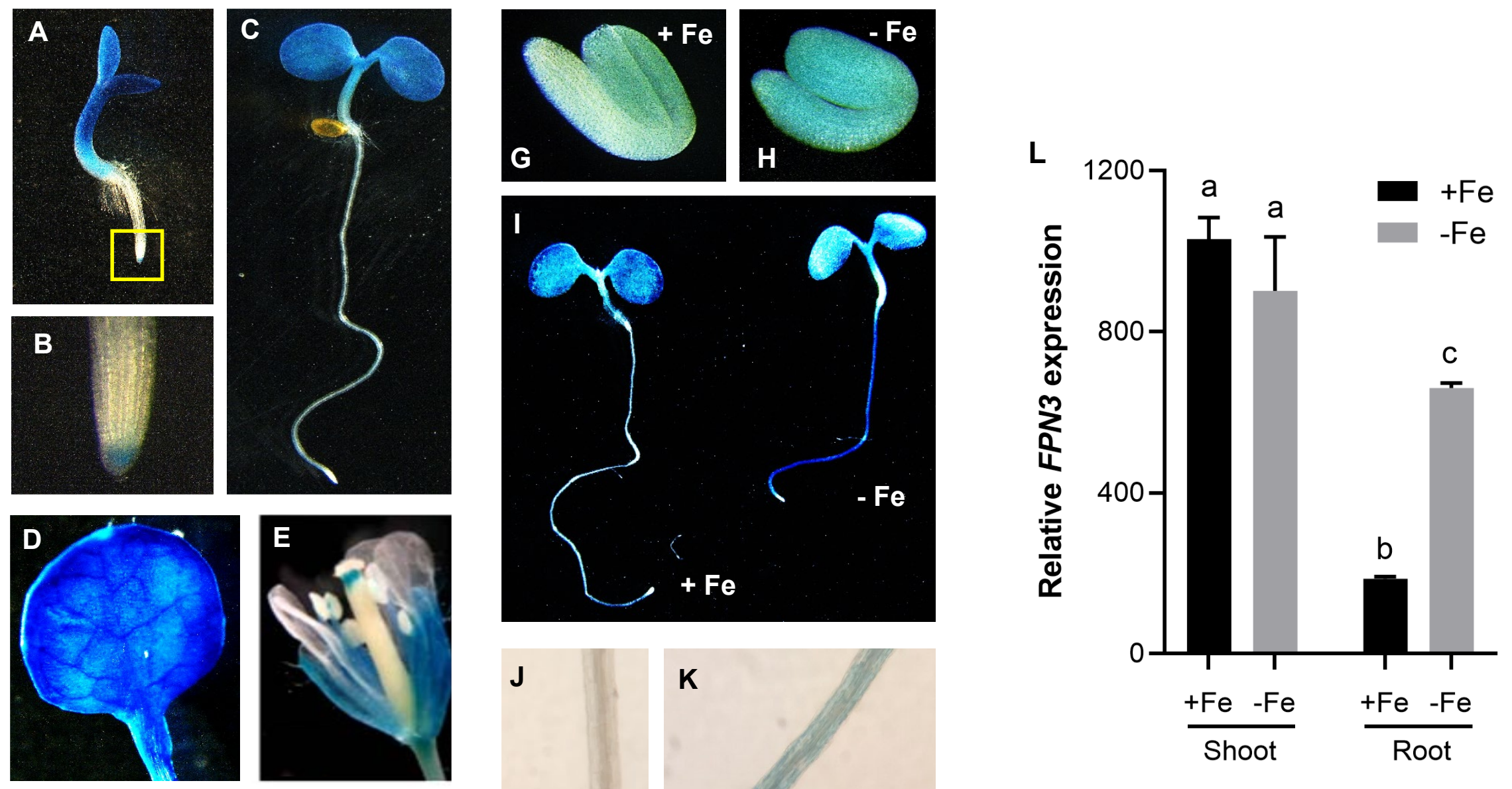

F

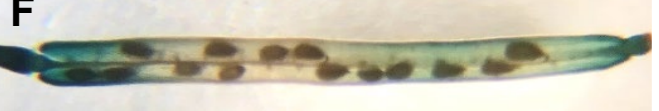


Figure 4.
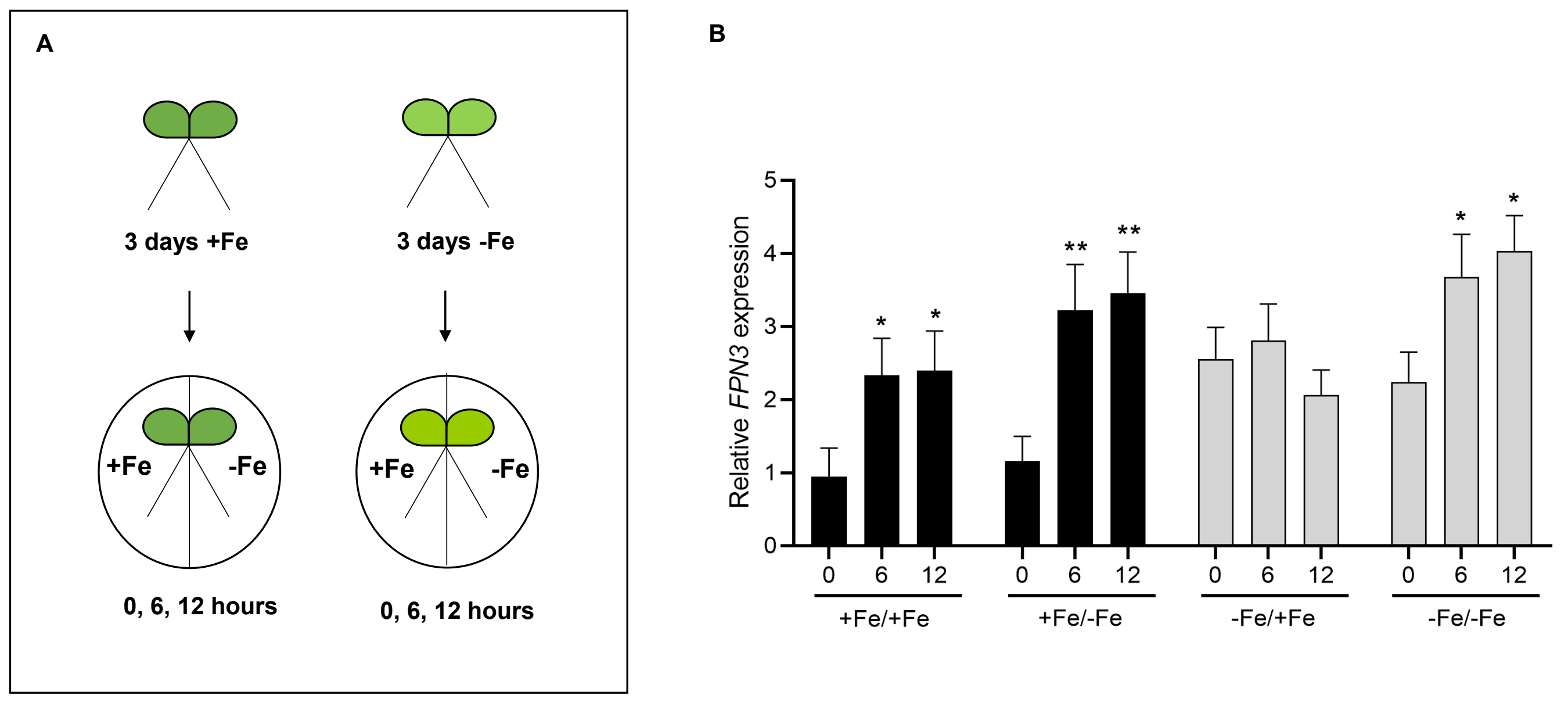
Figure 5.
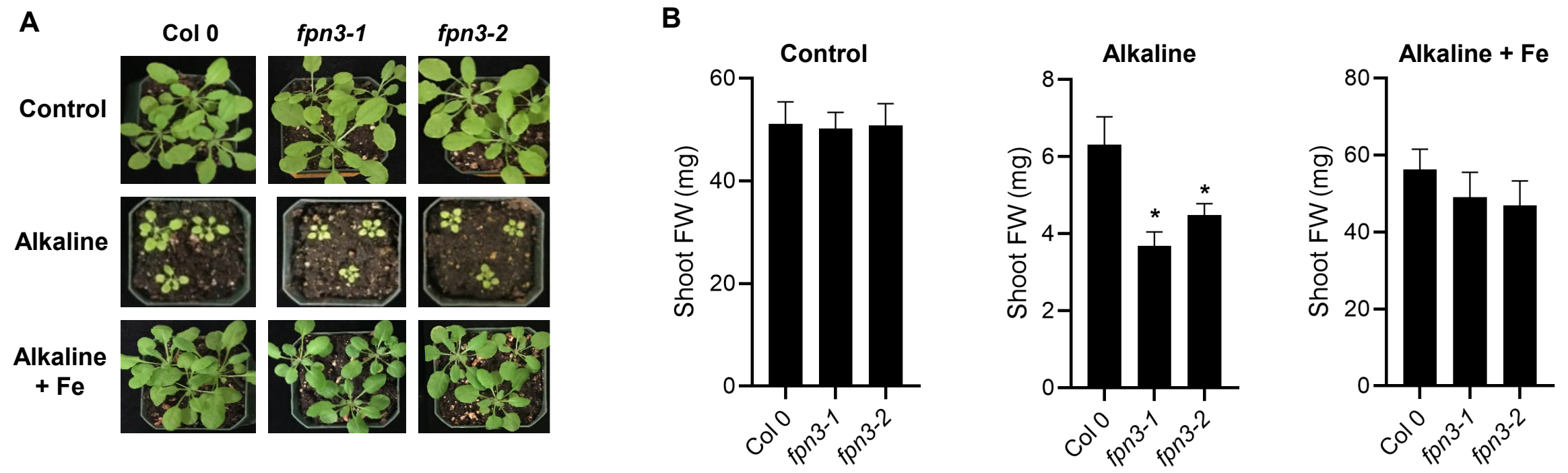
Figure 6.
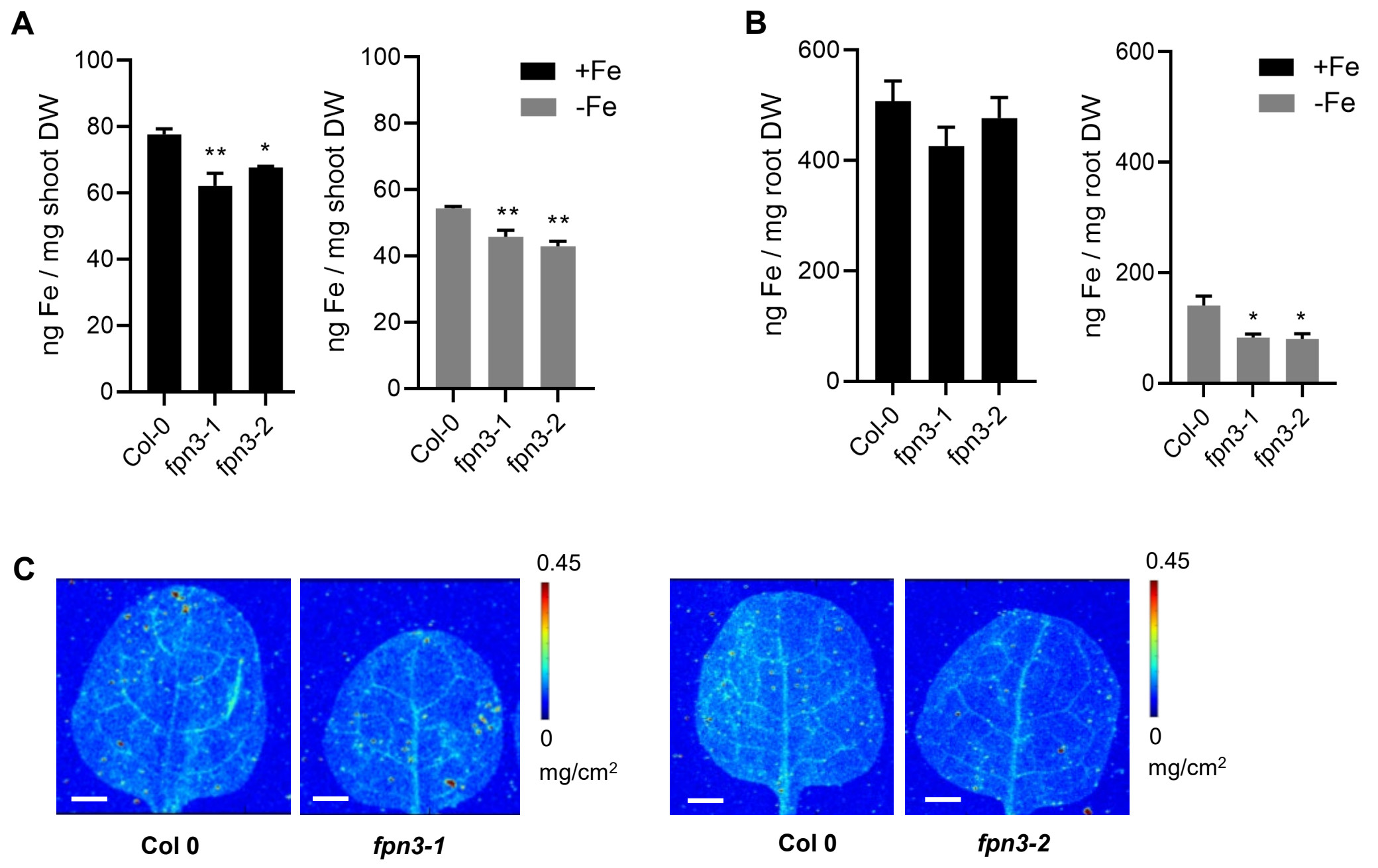
Figure 7.
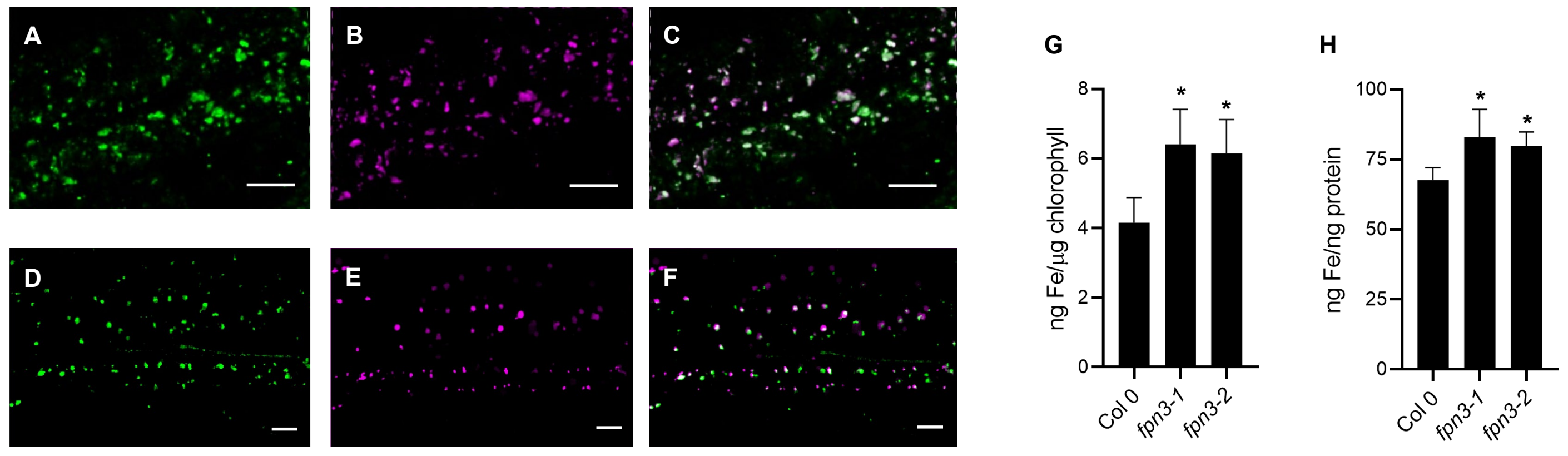
Figure 8.
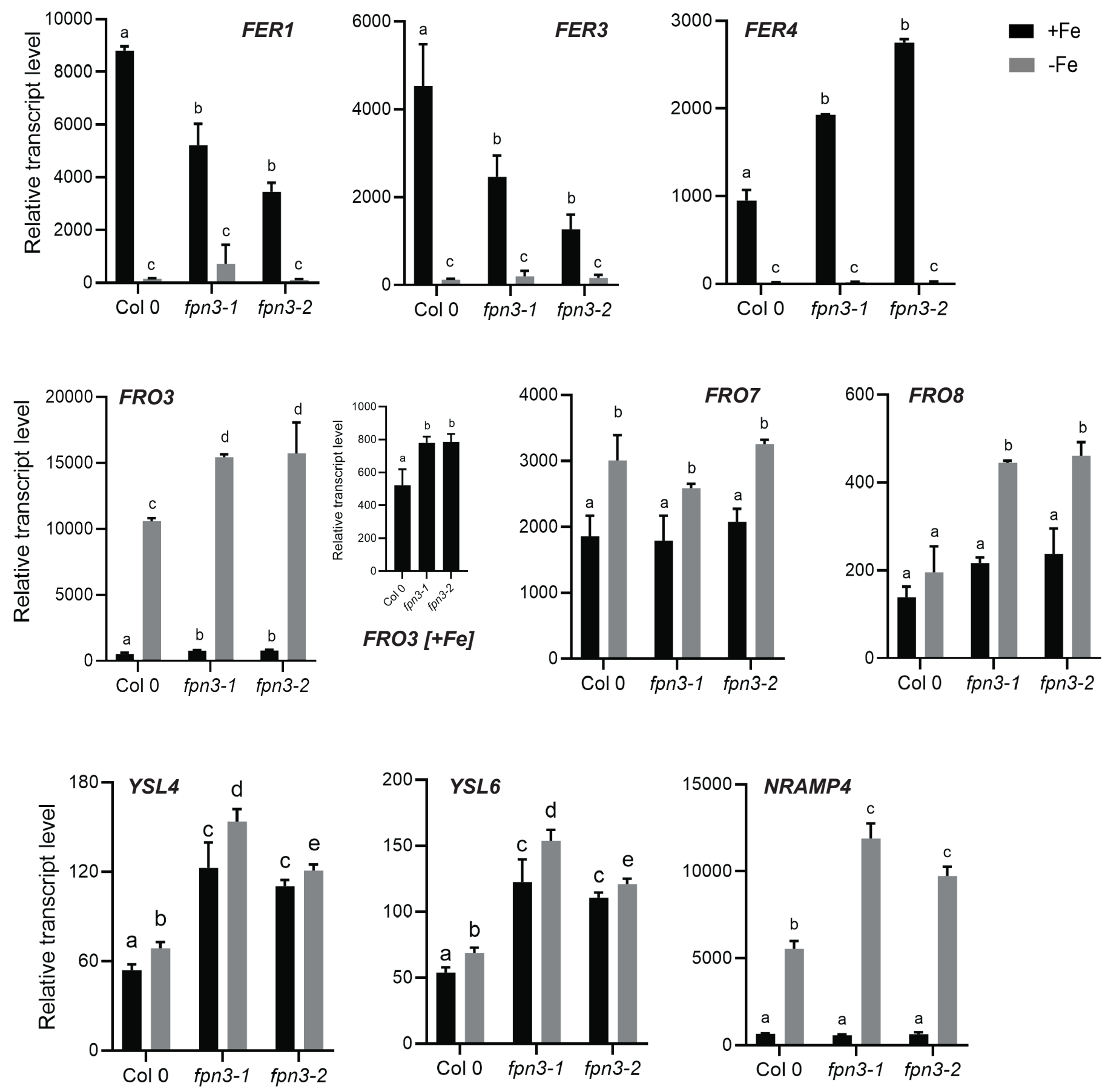
bioRxiv preprint doi: https://doi.org/10.1101/2020.07.15.203646; this version posted November 7,2020. The copyright holder for this preprint (which was not certified by peer review) is the author/funder, who has granted bioRxiv a license to display the preprint in perpetuity. It is made Figure 9. available under aCC-BY 4.0 International license.
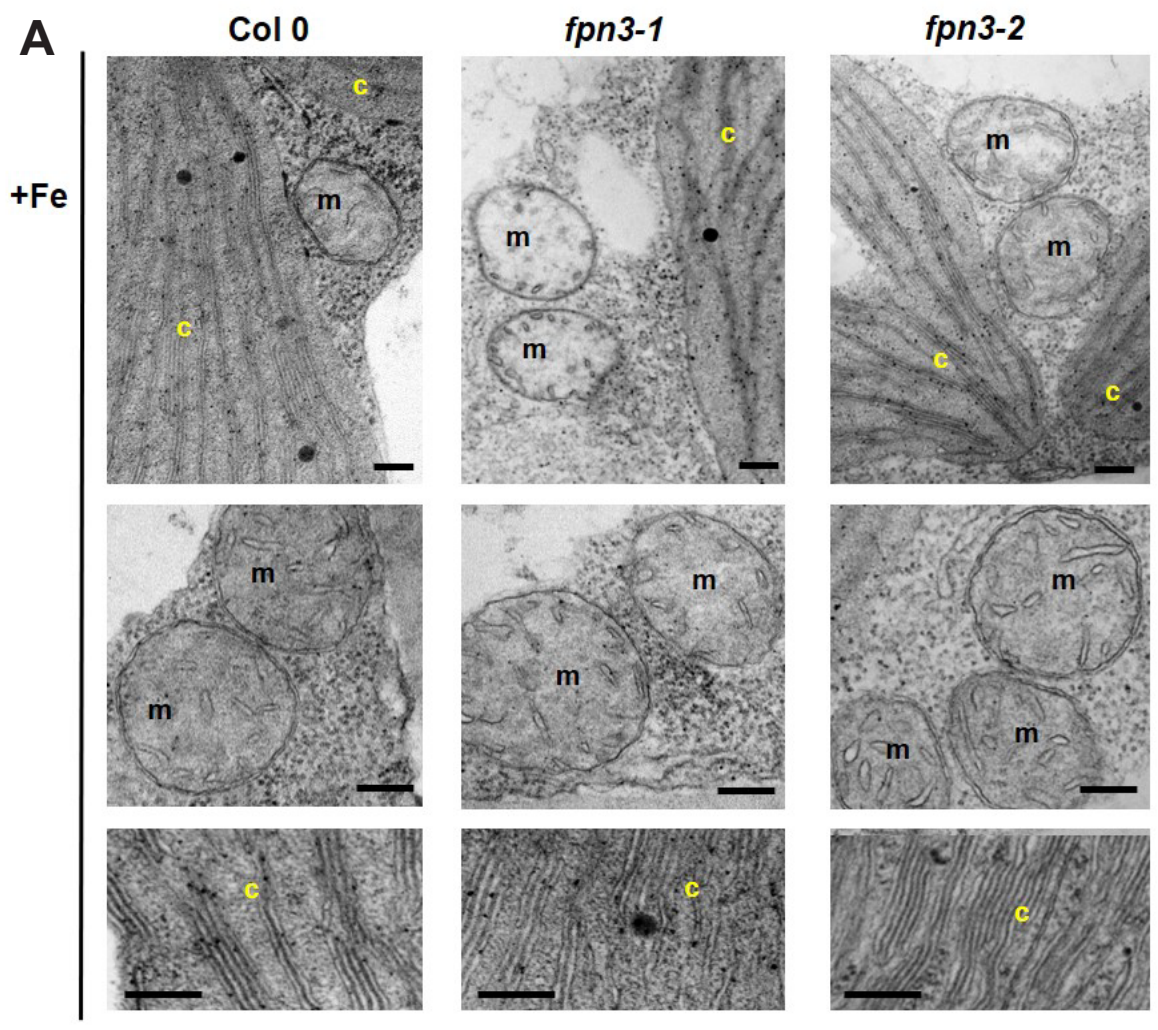

\section{B}
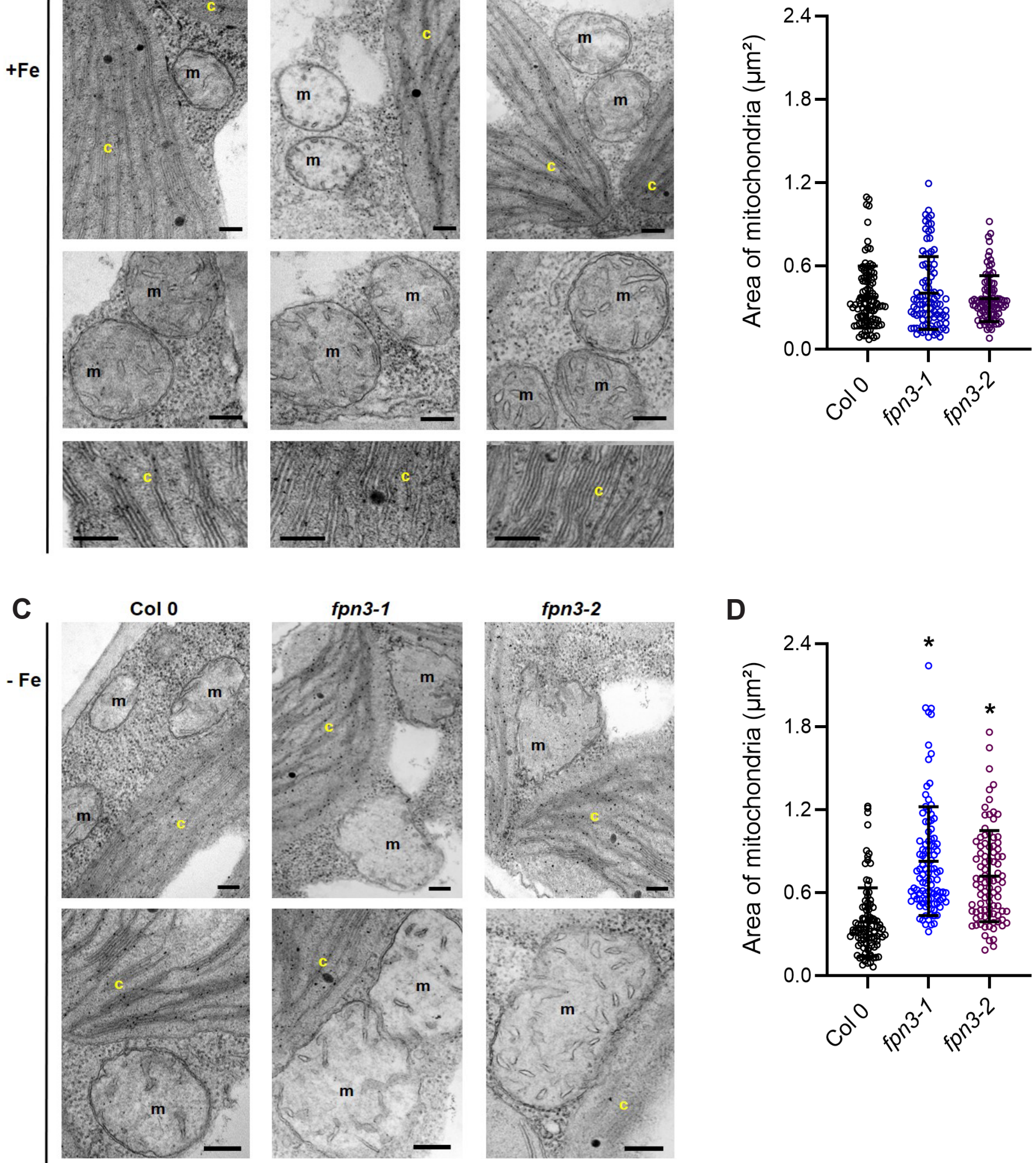

D
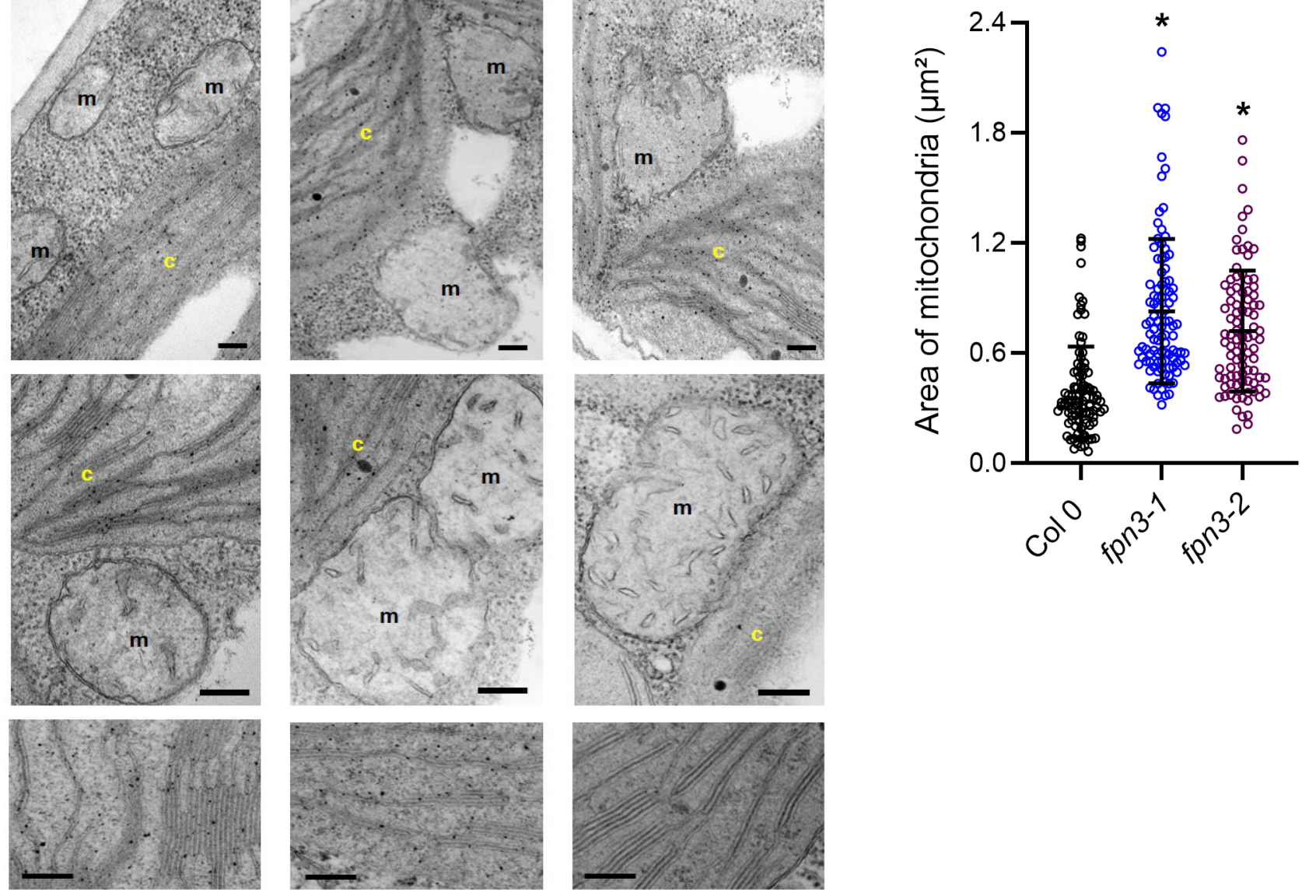